\title{
From loops to trees by-passing Feynman's theorem
}

\author{
Stefano Catani ${ }^{(a) *}$, Tanju Gleisberg ${ }^{(b) \dagger}$, Frank Krauss ${ }^{(c) \ddagger}$, \\ Germán Rodrigo $^{(d) \S}$ and Jan-Christopher Winter ${ }^{(e)}$ ฯ \\ ${ }^{(a)}$ INFN, Sezione di Firenze and Dipartimento di Fisica, Università di Firenze, \\ I-50019 Sesto Fiorentino, Florence, Italy \\ (b) Stanford Linear Accelerator Center, Stanford University \\ Stanford, CA 94309, USA \\ (c) Institute for Particle Physics Phenomenology, Durham University, \\ Durham DH1 3LE, UK \\ ${ }^{(d)}$ Instituto de Física Corpuscular, CSIC-Universitat de València, \\ Apartado de Correos 22085, E-46071 Valencia, Spain \\ ${ }^{(e)}$ Fermi National Accelerator Laboratory, Batavia, IL 60510, USA
}

\begin{abstract}
We derive a duality relation between one-loop integrals and phase-space integrals emerging from them through single cuts. The duality relation is realized by a modification of the customary $+i 0$ prescription of the Feynman propagators. The new prescription regularizing the propagators, which we write in a Lorentz covariant form, compensates for the absence of multiplecut contributions that appear in the Feynman Tree Theorem. The duality relation can be applied to generic one-loop quantities in any relativistic, local and unitary field theories. It is suitable for applications to the analytical calculation of one-loop scattering amplitudes, and to the numerical evaluation of cross-sections at next-to-leading order.
\end{abstract}

arXiv:0804.xxxx [hep-ph]

20 April 2008

\footnotetext{
*E-mail: stefano.catani@fi.infn.it

${ }^{\dagger}$ E-mail: tanju@slac.stanford.edu

${ }_{\ddagger}^{\ddagger}$ E-mail: frank.krauss@durham.ac.uk

$\S$ E-mail: german.rodrigo@ific.uv.es

๑E-mail: jwinter@fnal.gov
} 


\section{Introduction}

The Feynman Tree Theorem (FTT) [1, 2] applies to any (local and unitary) quantum field theories in Minkowsky space with an arbitrary number $d$ of space-time dimensions. It relates perturbative scattering amplitudes and Green's functions at the loop level with analogous quantities at the tree level. This relation follows from a basic and more elementary relation between loop integrals and phase-space integrals. Using this basic relation loop Feynman diagrams can be rewritten in terms of phase-space integrals of tree-level Feynman diagrams. The corresponding tree-level Feynman diagrams are then obtained by considering multiple cuts (single cuts, double cuts, triple cuts and so forth) of the original loop Feynman diagram.

We have recently proposed a method $[3,4,5]$ to numerically compute multi-leg one-loop cross sections in perturbative field theories. The starting point of this method is a duality relation between one-loop integrals and phase-space integrals. Although the analogy with the FTT is quite close, there are important differences. The key difference is that the duality relation involves only single cuts of the one-loop Feynman diagrams. Both the FTT and the duality relation can be derived by using the residue theorem*.

In this paper, we illustrate and derive the duality relation. Since the FTT has recently attracted a renewed interest [6] in the context of twistor-inspired methods [7, 8] to evaluate one-loop scattering amplitudes [9], we also discuss its correspondence (including similarities and differences) with the duality relation.

The outline of the paper is as follows. In Section 2, we introduce our notation. In Section 3, we briefly recall how the FTT relates one-loop integrals with multiple-cut phasespace integrals. In Section 4, we present one of the main results of this publication: we derive and illustrate the duality relation between one-loop integrals and single-cut phasespace integrals. We also prove that the duality relation requires to properly regularize propagators by a complex Lorentz-covariant prescription, which is different from the customary $+i 0$ prescription of the Feynman propagators. The duality is illustrated in Section 5 by considering the two-point function as the simplest example application. The correspondence between the FTT and the duality relation is formalized in Section 6. In Section 7, we explore the one-to-one correspondence between one-loop Feynman integrals and single-cut integrals on more mathematical grounds, and establish a generalized duality relation. The treatment of particle masses (including complex masses of unstable particles) when cutting loop integrals is discussed in Section 8. In Section 9, we analyze the effect of the gauge poles introduced by the propagators of the gauge fields in local gauge theories. In Section 10, we discuss the extension of the duality relation to one-loop Green's functions and scattering amplitudes. Some final remarks are presented in Section 11. Details about the derivation of the duality relation by using the residue theorem are discussed in Appendix A. The proof of an algebraic relation is presented in Appendix B.

*Within the context of loop integrals, the use of the residue theorem has been considered many times in textbooks and in the literature. 


\section{Notation}

The FTT and the duality relation can be illustrated with no loss of generality by considering their application to the basic ingredient of any one-loop Feynman diagrams, namely a generic one-loop scalar integral $L^{(N)}$ with $N(N \geq 2)$ external legs.

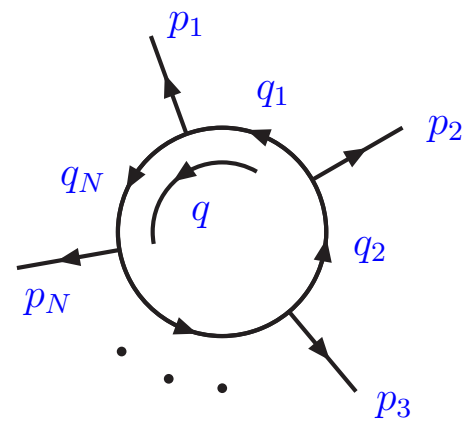

Figure 1: Momentum configuration of the one-loop N-point scalar integral.

The momenta of the external legs are denoted by $p_{1}^{\mu}, p_{2}^{\mu}, \ldots, p_{N}^{\mu}$ and are clockwise ordered (Fig. 1). All are taken as outgoing. To simplify the notation and the presentation, we also limit ourselves in the beginning to considering massless internal lines only. Thus, the one-loop integral $L^{(N)}$ can in general be expressed as:

$$
L^{(N)}\left(p_{1}, p_{2}, \ldots, p_{N}\right)=-i \int \frac{d^{d} q}{(2 \pi)^{d}} \prod_{i=1}^{N} \frac{1}{q_{i}^{2}+i 0},
$$

where $q^{\mu}$ is the loop momentum (which flows anti-clockwise). The momenta of the internal lines are denoted by $q_{i}^{\mu}$; they are given by

$$
q_{i}=q+\sum_{k=1}^{i} p_{k}
$$

and momentum conservation results in the constraint

$$
\sum_{i=1}^{N} p_{i}=0
$$

The value of the label $i$ of the external momenta is defined modulo $N$, i.e. $p_{N+i} \equiv p_{i}$.

The number of space-time dimensions is denoted by $d$ (the convention for the Lorentzindices adopted here is $\mu=0,1, \ldots, d-1)$ with metric tensor $g^{\mu \nu}=\operatorname{diag}(+1,-1, \ldots,-1)$. Note that $d$ does not necessarily have integer value, but it must satisfy $d \geq 1$ (as in the case of loop integrals in dimensional regularization). The space-time coordinates of any momentum $k_{\mu}$ are denoted as $k_{\mu}=\left(k_{0}, \mathbf{k}\right)$, where $k_{0}$ is the energy (time component) of $k_{\mu}$. It is also convenient to introduce light-cone coordinates $k_{\mu}=\left(k_{+}, \mathbf{k}_{\perp}, k_{-}\right)$, where $k_{ \pm}=\left(k_{0} \pm k_{d-1}\right) / \sqrt{2}$.

Throughout the paper we use the following shorthand notation:

$$
-i \int \frac{d^{d} q}{(2 \pi)^{d}} \cdots \equiv \int_{q} \cdots
$$


When we factorize off in a loop integral the integration over the momentum coordinate $q_{0}$ or $q_{+}$, we write

$$
-i \int_{-\infty}^{+\infty} d q_{0} \int \frac{d^{d-1} \mathbf{q}}{(2 \pi)^{d}} \cdots \equiv \int d q_{0} \int_{\mathbf{q}} \cdots
$$

and

$$
-i \int_{-\infty}^{+\infty} d q_{+} \int_{-\infty}^{+\infty} d q_{-} \int \frac{d^{d-2} \mathbf{q}_{\perp}}{(2 \pi)^{d}} \cdots \equiv \int d q_{+} \int_{\left(q_{-}, \mathbf{q}_{\perp}\right)} \cdots
$$

respectively. The customary phase-space integral of a physical massless particle with momentum $q$ (i.e. an on-shell particle with positive-definite energy: $q^{2}=0, q_{0} \geq 0$ ) reads

$$
\int \frac{d^{d} q}{(2 \pi)^{d-1}} \theta\left(q_{0}\right) \delta\left(q^{2}\right) \cdots \equiv \int_{q} \widetilde{\delta}(q) \cdots
$$

where we have defined

$$
\widetilde{\delta}(q) \equiv 2 \pi i \theta\left(q_{0}\right) \delta\left(q^{2}\right)=2 \pi i \delta_{+}\left(q^{2}\right)
$$

Using this shorthand notation, the one-loop integral $L^{(N)}$ in Eq. (1) can be cast into

$$
L^{(N)}\left(p_{1}, p_{2}, \ldots, p_{N}\right)=\int_{q} \prod_{i=1}^{N} G\left(q_{i}\right)
$$

where $G(q)$ denotes the customary Feynman propagator,

$$
G(q) \equiv \frac{1}{q^{2}+i 0}
$$

We also introduce the advanced propagator $G_{A}(q)$,

$$
G_{A}(q) \equiv \frac{1}{q^{2}-i 0 q_{0}}
$$

We recall that the Feynman and advanced propagators only differ in the position of the particle poles in the complex plane (Fig. 2). Using $q^{2}=q_{0}^{2}-\mathbf{q}^{2}=2 q_{+} q_{-}-\mathbf{q}_{\perp}^{2}$, we therefore have

$$
[G(q)]^{-1}=0 \quad \Longrightarrow \quad q_{0}= \pm \sqrt{\mathbf{q}^{2}-i 0}, \text { or } q_{ \pm}=\frac{\mathbf{q}_{\perp}^{2}-i 0}{2 q_{\mp}}
$$

and

$$
\left[G_{A}(q)\right]^{-1}=0 \quad \Longrightarrow \quad q_{0} \simeq \pm \sqrt{\mathbf{q}^{2}}+i 0 \quad \text { or } q_{ \pm} \simeq \frac{\mathbf{q}_{\perp}^{2}}{2 q_{\mp}}+i 0
$$

Thus, in the complex plane of the variable $q_{0}$ (or, equivalently ${ }^{\dagger}, q_{ \pm}$), the pole with positive (negative) energy of the Feynman propagator is slightly displaced below (above) the real axis, while both poles (independently of the sign of the energy) of the advanced propagator are slightly displaced above the real axis.

\footnotetext{
${ }^{\dagger}$ To be precise, each propagator leads to two poles in the plane $q_{0}$ and to only one pole in the plane $q_{+}$ (or $q_{-}$).
} 

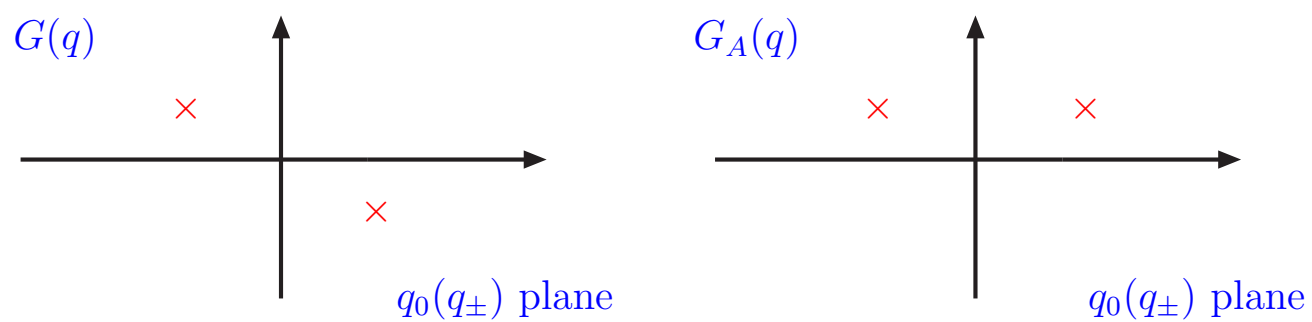

Figure 2: Location of the particle poles of the Feynman (left) and advanced (right) propagators, $G(q)$ and $G_{A}(q)$, in the complex plane of the variable $q_{0}$ or $q_{ \pm}$.

\section{The Feynman theorem}

In this Section we briefly recall the FTT $[1,2]$.

To this end, we first introduce the advanced one-loop integral $L_{A}^{(N)}$, which is obtained from $L^{(N)}$ in Eq. (9) by replacing the Feynman propagators $G\left(q_{i}\right)$ with the corresponding advanced propagators $G_{A}\left(q_{i}\right)$ :

$$
L_{A}^{(N)}\left(p_{1}, p_{2}, \ldots, p_{N}\right)=\int_{q} \prod_{i=1}^{N} G_{A}\left(q_{i}\right) .
$$

Then, we note that

$$
L_{A}^{(N)}\left(p_{1}, p_{2}, \ldots, p_{N}\right)=0
$$

The proof of Eq. (15) can be carried out in an elementary way by using the Cauchy residue theorem and choosing a suitable integration path $C_{L}$. We have

$$
\begin{aligned}
& L_{A}^{(N)}\left(p_{1}, p_{2}, \ldots, p_{N}\right)=\int_{\mathbf{q}} \int d q_{0} \prod_{i=1}^{N} G_{A}\left(q_{i}\right) \\
& =\int_{\mathbf{q}} \int_{C_{L}} d q_{0} \prod_{i=1}^{N} G_{A}\left(q_{i}\right)=-2 \pi i \int_{\mathbf{q}} \sum \operatorname{Res}_{\left\{\operatorname{Im} q_{0}<0\right\}}\left[\prod_{i=1}^{N} G_{A}\left(q_{i}\right)\right]=0 .
\end{aligned}
$$

The loop integral is evaluated by integrating first over the energy component $q_{0}$. Since the integrand is convergent when $q_{0} \rightarrow \infty$, the $q_{0}$ integration can be performed along the contour $C_{L}$, which is closed at $\infty$ in the lower half-plane of the complex variable $q_{0}$ (Fig. 3left). The only singularities of the integrand with respect to the variable $q_{0}$ are the poles of the advanced propagators $G_{A}\left(q_{i}\right)$, which are located in the upper half-plane. The integral along $C_{L}$ is then equal to the sum of the residues at the poles in the lower half-plane and therefore vanishes.

The advanced and Feynman propagators are related by

$$
G_{A}(q)=G(q)+\widetilde{\delta}(q)
$$



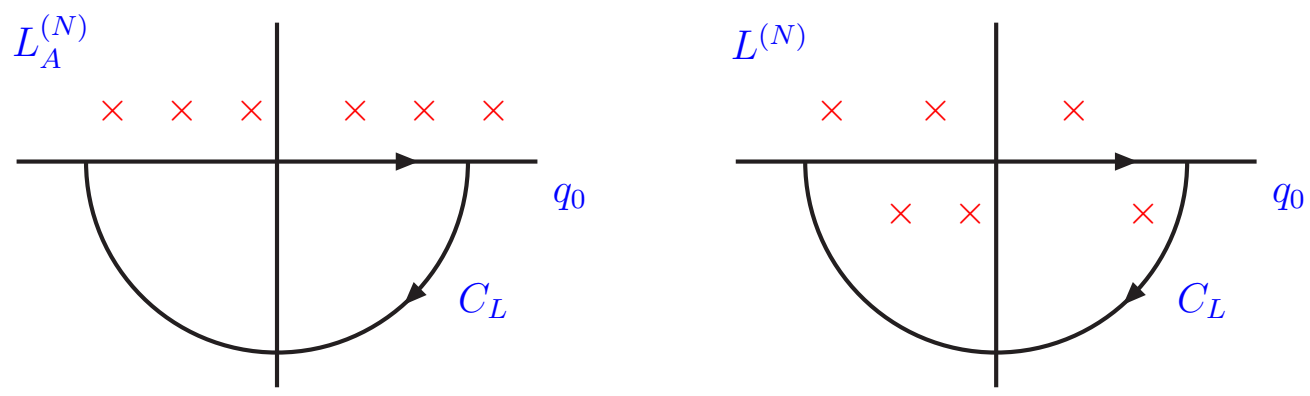

Figure 3: Location of poles and integration contour $C_{L}$ in the complex $q_{0}$-plane for the advanced (left) and Feynman (right) one-loop integrals, $L_{A}^{(N)}$ and $L^{(N)}$.

which can straightforwardly be obtained by using the elementary identity

$$
\frac{1}{x \pm i 0}=\mathrm{PV}\left(\frac{1}{x}\right) \mp i \pi \delta(x)
$$

where PV denotes the principal-value prescription. Inserting Eq. (17) into the right-hand side of Eq. (14) and collecting the contributions with an equal number of factors $G\left(q_{i}\right)$ and $\widetilde{\delta}\left(q_{j}\right)$, we obtain a relation between $L_{A}^{(N)}$ and the one-loop integral $L^{(N)}$ :

$$
\begin{aligned}
& L_{A}^{(N)}\left(p_{1}, p_{2}, \ldots, p_{N}\right)=\int_{q} \prod_{i=1}^{N}\left[G\left(q_{i}\right)+\widetilde{\delta}\left(q_{i}\right)\right] \\
& =L^{(N)}\left(p_{1}, p_{2}, \ldots, p_{N}\right)+L_{1-\text { cut }}^{(N)}\left(p_{1}, p_{2}, \ldots, p_{N}\right)+\cdots+L_{\mathrm{N}-\text { cut }}^{(N)}\left(p_{1}, p_{2}, \ldots, p_{N}\right) .
\end{aligned}
$$

Here, the single-cut contribution is given by

$$
L_{1-\text { cut }}^{(N)}\left(p_{1}, p_{2}, \ldots, p_{N}\right)=\int_{q} \sum_{i=1}^{N} \widetilde{\delta}\left(q_{i}\right) \prod_{\substack{j=1 \\ j \neq i}}^{N} G\left(q_{j}\right) .
$$

In general, the $m$-cut terms $L_{\mathrm{m}-\text { cut }}^{(N)}(m \leq N)$ are the contributions with precisely $m$ delta functions $\widetilde{\delta}\left(q_{i}\right)$ :

$$
L_{\mathrm{m}-\text { cut }}^{(N)}\left(p_{1}, p_{2}, \ldots, p_{N}\right)=\int_{q}\left\{\widetilde{\delta}\left(q_{1}\right) \ldots \widetilde{\delta}\left(q_{m}\right) G\left(q_{m+1}\right) \ldots G\left(q_{N}\right)+\text { uneq. perms. }\right\},
$$

where the sum in the curly bracket includes all the permutations of $q_{1}, \ldots, q_{N}$ that give unequal terms in the integrand.

Recalling that $L_{A}^{(N)}$ vanishes, cf. Eq. (15), Eq. (19) results in:

$$
L^{(N)}\left(p_{1}, p_{2}, \ldots, p_{N}\right)=-\left[L_{1-\text { cut }}^{(N)}\left(p_{1}, p_{2}, \ldots, p_{N}\right)+\cdots+L_{\mathrm{N}-\text { cut }}^{(N)}\left(p_{1}, p_{2}, \ldots, p_{N}\right)\right] .
$$

This equation is the FTT in the specific case of the one-loop integral $L^{(N)}$. The FTT relates the one-loop integral $L^{(N)}$ to the multiple-cut ${ }^{\ddagger}$ integrals $L_{\mathrm{m} \text {-cut }}^{(N)}$. Each delta function $\widetilde{\delta}\left(q_{i}\right)$

\footnotetext{
${ }^{\ddagger}$ If the number of space-time dimensions is $d$, the right-hand side of Eq. (22) receives contributions only from the terms with $m \leq d$; the terms with larger values of $m$ vanish, since the corresponding number of delta functions in the integrand is larger than the number of integration variables.
} 
in $L_{\mathrm{m}-\text { cut }}^{(N)}$ replaces the corresponding Feynman propagator in $L^{(N)}$ by cutting the internal line with momentum $q_{i}$. This is synonymous to setting the respective particle on shell. An $m$-particle cut decomposes the one-loop diagram in $m$ tree diagrams: in this sense, the FTT allows us to calculate loop-level diagrams from tree-level diagrams.
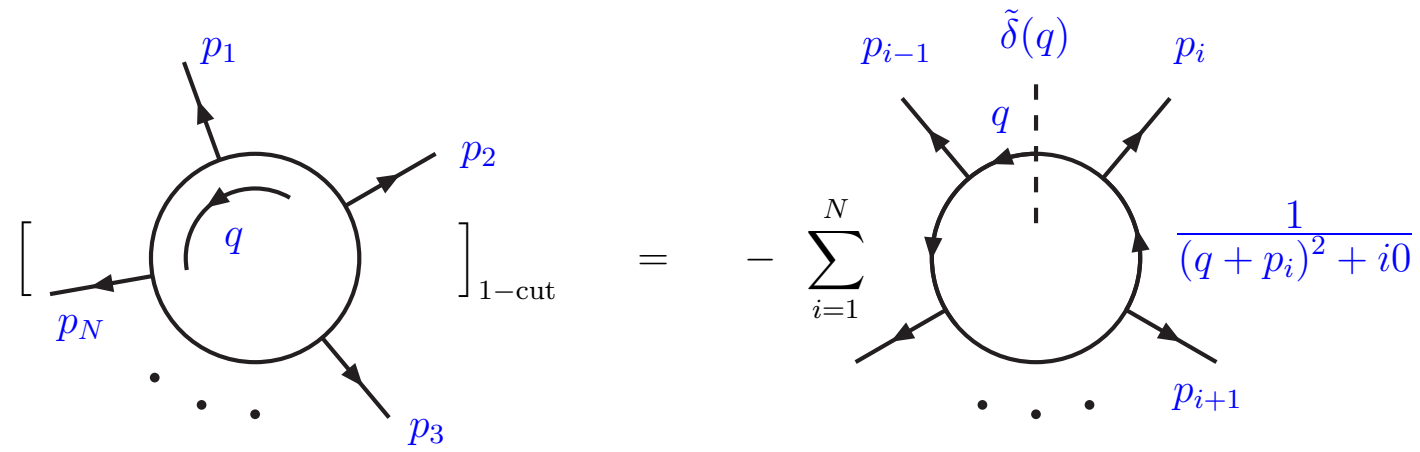

Figure 4: The single-cut contribution of the Feynman Tree Theorem to the one-loop $N$ point scalar integral. Graphical representation as a sum of $N$ basic single-cut phase-space integrals.

In view of the discussion in the following sections, it is useful to consider the single-cut contribution $L_{1-\text { cut }}^{(N)}$ on the right-hand side of Eq. (22). In the case of single-cut contributions, the FTT replaces the one-loop integral with the customary one-particle phase-space integral, see Eqs. (7) and (20). Using the invariance of the loop-integration measure under translations of the loop momentum $q$, we can perform the momentum shift $q \rightarrow q-\sum_{k=1}^{i} p_{k}$ in the term proportional to $\widetilde{\delta}\left(q_{i}\right)$ on the right-hand side of Eq. (20). Thus, cf. Eq. (2), we have $q_{i} \rightarrow q$ and $q_{j} \rightarrow q+\left(p_{i+1}+p_{i+2}+\cdots+p_{i+j}\right)$, with $i \neq j$. We can repeat the same shift for each of the terms $(i=1,2, \ldots, N)$ in the sum on the right-hand side of Eq. (20), and we can rewrite $L_{1-\text { cut }}^{(N)}$ as a sum of $N$ basic phase-space integrals (Fig. 4):

$$
\begin{aligned}
L_{1-\text { cut }}^{(N)}\left(p_{1}, p_{2}, \ldots, p_{N}\right) & =I_{1-\text { cut }}^{(N-1)}\left(p_{1}, p_{1}+p_{2}, \ldots, p_{1}+p_{2}+\cdots+p_{N-1}\right)+\text { cyclic perms. } \\
& =\sum_{i=1}^{N} I_{1-\text { cut }}^{(N-1)}\left(p_{i}, p_{i}+p_{i+1}, \ldots, p_{i}+p_{i+1}+\cdots+p_{i+N-1}\right)
\end{aligned}
$$

We denote the basic one-particle phase-space integrals with $n$ Feynman propagators by $I_{1-\text { cut }}^{(n)}$. They are defined as follows:

$$
I_{1-\text { cut }}^{(n)}\left(k_{1}, k_{2}, \ldots, k_{n}\right)=\int_{q} \widetilde{\delta}(q) \prod_{j=1}^{n} G\left(q+k_{j}\right)=\int_{q} \widetilde{\delta}(q) \prod_{j=1}^{n} \frac{1}{2 q k_{j}+k_{j}^{2}+i 0} .
$$

The extension of the FTT from the one-loop integrals $L^{(N)}$ to one-loop scattering amplitudes $\mathcal{A}^{\text {(1-loop) }}$ (or Green's functions) in perturbative field theories is straightforward, provided the corresponding field theory is unitary and local. The generalization of Eq. (22) to arbitrary scattering amplitudes is $[1,2]$ :

$$
\mathcal{A}^{(1-\mathrm{loop})}=-\left[\mathcal{A}_{1-\mathrm{cut}}^{(1-\mathrm{loop})}+\mathcal{A}_{2-\text { cut }}^{(1-\mathrm{loop})}+\ldots\right]
$$


where $\mathcal{A}_{\mathrm{m} \text {-cut }}^{(1 \text {-loop })}$ is obtained in the same way as $L_{\mathrm{m} \text {-cut }}^{(N)}$, i.e. by starting from $\mathcal{A}^{(1-\text { loop })}$ and considering all possible replacements of $m$ Feynman propagators $G\left(q_{i}\right)$ of its loop internal lines with the 'cut propagators' $\widetilde{\delta}\left(q_{i}\right)$.

The proof of Eq. (25) directly follows from Eq. (22): $\mathcal{A}^{(1-\text { loop) }}$ is a linear combination of one-loop integrals that differ from $L^{(N)}$ only by the inclusion of interaction vertices and, eventually, particle masses. As briefly recalled below, these differences have harmless consequences on the derivation of the FTT.

Including particle masses in the advanced and Feynman propagators has an effect on the location of the poles produced by the internal lines in the loop. However, as long as the masses are real, as in the case of unitary theories, the position of the poles in the complex plane of the variable $q_{0}$ is affected only by a translation parallel to the real axis, with no effect on the imaginary part of the poles. This translation does not interfere with the proof of the FTT as given in Eqs. (14)-(22). Therefore, the effect of a particle mass $M_{i}$ in a loop internal line with momentum $q_{i}$ simply amounts to modifying the corresponding on-shell delta function $\widetilde{\delta}\left(q_{i}\right)$ when this line is cut to obtain $\mathcal{A}_{\mathrm{m}-\text { cut }}^{(1-\text { loop }}$. This modification then leads to the obvious replacement:

$$
\widetilde{\delta}\left(q_{i}\right) \rightarrow \widetilde{\delta}\left(q_{i} ; M_{i}\right)=2 \pi i \theta\left(q_{i 0}\right) \delta\left(q_{i}^{2}-M_{i}^{2}\right)=2 \pi i \delta_{+}\left(q_{i}^{2}-M_{i}^{2}\right)
$$

Including interaction vertices has the effect of introducing numerator factors in the integrand of the one-loop integrals. As long as the theory is local, these numerator factors are at worst polynomials of the integration momentum $q \S$. In the complex plane of the variable $q_{0}$, this polynomial behavior does not lead to additional singularities at any finite values of $q_{0}$. The only danger, when using the Cauchy theorem as in Eq. (16) to prove the FTT, stems from polynomials of high degree that can spoil the convergence of the $q_{0}$-integration at infinity. Nonetheless, if the field theory is unitary, these singularities at infinity never occur since the degree of the polynomials in the various integrands is always sufficiently limited by the unitarity constraint.

\section{A duality theorem}

In this Section we derive and illustrate the duality relation between one-loop integrals and single-cut phase-space integrals. This relation is the main general result of the present work.

Rather than starting from $L_{A}^{(N)}$, we directly apply the residue theorem to the compu-

\footnotetext{
$\S$ This statement is not completely true in the case of gauge theories and, in particular, in the case of gauge-dependent quantities. The discussion of the additional issues that arise in gauge theories is postponed to Sect. 9 .
} 
tation of $L^{(N)}$. We proceed exactly as in Eq. (16), and obtain

$$
\begin{aligned}
& L^{(N)}\left(p_{1}, p_{2}, \ldots, p_{N}\right)=\int_{\mathbf{q}} \int d q_{0} \prod_{i=1}^{N} G\left(q_{i}\right) \\
& =\int_{\mathbf{q}} \int_{C_{L}} d q_{0} \prod_{i=1}^{N} G\left(q_{i}\right)=-2 \pi i \int_{\mathbf{q}} \sum \operatorname{Res}_{\left\{\operatorname{Im} q_{0}<0\right\}}\left[\prod_{i=1}^{N} G\left(q_{i}\right)\right] .
\end{aligned}
$$

At variance with $G_{A}\left(q_{i}\right)$, each of the Feynman propagators $G\left(q_{i}\right)$ has single poles in both the upper and lower half-planes of the complex variable $q_{0}$ (see Fig. 3-right) and therefore the integral does not vanish as in the case of the advanced propagators. In contrast, here, the $N$ poles in the lower half-plane contribute to the residues in Eq. (27).

The calculation of these residues is elementary, but it involves several subtleties. The detailed calculation, including a discussion of its subtle points, is presented in Appendix A. In the present Section we limit ourselves to sketching the derivation of the result of this computation.

The sum over residues in Eq. (27) receives contributions from $N$ terms, namely the $N$ residues at the poles with negative imaginary part of each of the propagators $G\left(q_{i}\right)$, with $i=1, \ldots, N$, see Eq. (12). Considering the residue at the $i$-th pole we write

$$
\operatorname{Res}_{\{i-\text { th pole }\}}\left[\prod_{j=1}^{N} G\left(q_{j}\right)\right]=\left[\operatorname{Res}_{\{i-\text { th pole }\}} G\left(q_{i}\right)\right]\left[\prod_{\substack{j=1 \\ j \neq i}}^{N} G\left(q_{j}\right)\right]_{\{i-\text { th pole }\}},
$$

where we have used the fact that the propagators $G\left(q_{j}\right)$, with $j \neq i$, are not singular at the value of the pole of $G\left(q_{i}\right)$. Therefore, they can be directly evaluated at this value.

The calculation of the residue of $G\left(q_{i}\right)$ is straightforward and gives

$$
\left[\operatorname{Res}_{\{i-\text { th pole }\}} G\left(q_{i}\right)\right]=\left[\operatorname{Res}_{\{i-\text { th pole }\}} \frac{1}{q_{i}^{2}+i 0}\right]=\int d q_{0} \delta_{+}\left(q_{i}^{2}\right) .
$$

This result shows that considering the residue of the Feynman propagator of the internal line with momentum $q_{i}$ is equivalent to cut that line by including the corresponding on-shell propagator $\delta_{+}\left(q_{i}^{2}\right)$. In particular, the subscript + of $\delta_{+}$refers to the on-shell mode with positive definite energy, $q_{i 0}=\left|\mathbf{q}_{i}\right|$ : the positive-energy mode is selected by the Feynman $i 0$ prescription of the propagator $G\left(q_{i}\right)$. The insertion of Eq. (29) in Eq. (27) directly leads to a representation of the one-loop integral as a linear combination of $N$ single-cut phase-space integrals.

The calculation of the residue prefactor on the r.h.s. of Eq. (28) is more subtle (see Appendix A) and yields

$$
\left[\prod_{j \neq i} G\left(q_{j}\right)\right]_{\{i \text {-th pole }\}}=\left[\prod_{j \neq i} \frac{1}{q_{j}^{2}+i 0}\right]_{\{i-\text { th pole }\}}=\prod_{j \neq i} \frac{1}{q_{j}^{2}-i 0 \eta\left(q_{j}-q_{i}\right)}
$$

where $\eta$ is a future-like vector,

$$
\eta_{\mu}=\left(\eta_{0}, \eta\right), \quad \eta_{0} \geq 0, \eta^{2}=\eta_{\mu} \eta^{\mu} \geq 0
$$


i.e. a $d$-dimensional vector that can be either light-like $\left(\eta^{2}=0\right)$ or time-like $\left(\eta^{2}>0\right)$ with positive definite energy $\eta_{0}$. Note that the calculation of the residue at the pole of the internal line with momentum $q_{i}$ changes the propagators of the other lines in the loop integrals. Although the propagator of the $j$-th internal line has still the customary form $1 / q_{j}^{2}$, its singularity at $q_{j}^{2}=0$ is regularized by a different $i 0$ prescription: the original Feynman prescription $q_{j}^{2}+i 0$ is modified in the new prescription $q_{j}^{2}-i 0 \eta\left(q_{j}-q_{i}\right)$, which we name the 'dual' $i 0$ prescription or, briefly, the $\eta$ prescription. The dual $i 0$ prescription originates from the fact that the original Feynman propagator $1 /\left(q_{j}^{2}+i 0\right)$ is evaluated at the complex value of the loop momentum $q$ that is determined by the location of the pole at $q_{i}^{2}+i 0=0$. The $i 0$ dependence from the pole combines with the $i 0$ dependence in the Feynman propagator to obtain a total dependence as given by the dual $i 0$ prescription. The presence of the vector $\eta_{\mu}$ is due to the use of the residue theorem. To apply it to the calculation of the $d$ dimensional loop integral, we have to specify a system of coordinates (e.g. space-time or light-cone coordinates) and select one of them to be integrated over at fixed value of the remaining $d-1$ coordinates. Introducing the auxiliary vector $\eta_{\mu}$ with space-time coordinates $\eta_{\mu}=\left(\eta_{0}, \mathbf{0}_{\perp}, \eta_{d-1}\right)$, the selected system of coordinates can be denoted in a Lorentz-invariant form. Applying the residue theorem in the complex plane of the variable $q_{0}$ at fixed (and real) values of the coordinates $\mathbf{q}_{\perp}$ and $q_{d-1}^{\prime}=q_{d-1}-q_{0} \eta_{d-1} / \eta_{0}$ (to be precise, in Eq. (27) we actually used $\eta_{\mu}=(1,0)$ ), we obtain the result in Eq. (30).

The $\eta$ dependence of the ensuing $i 0$ prescription is thus a consequence of the fact that the residues at each of the poles are not Lorentz-invariant quantities. The Lorentz-invariance of the loop integral is recovered only after summing over all the residues.

Inserting the results of Eq. (28)-(30) in Eq. (27) we directly obtain the duality relation.
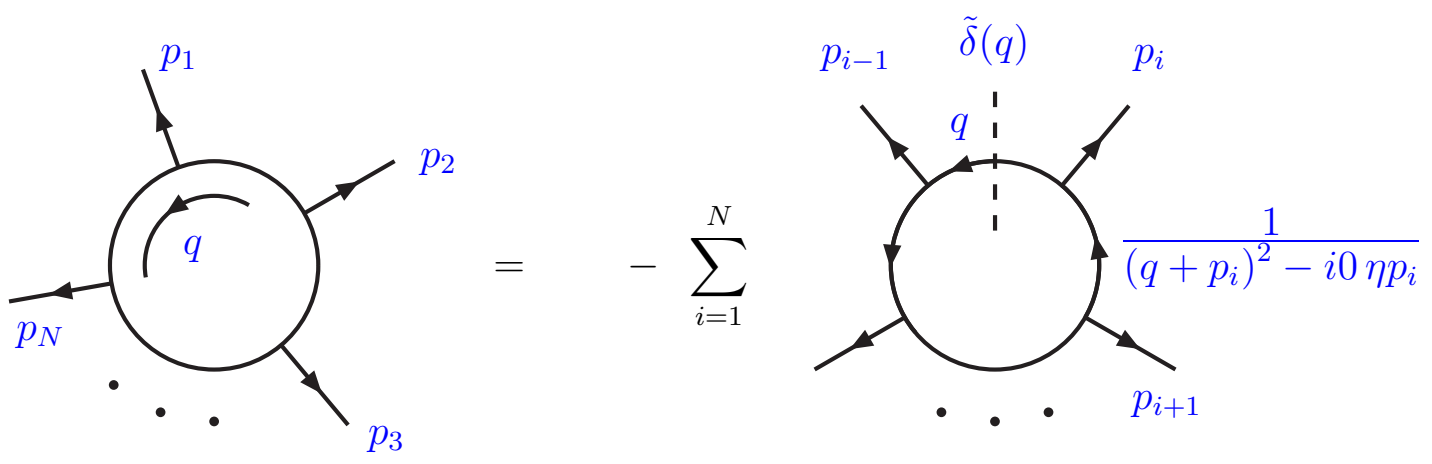

Figure 5: The duality relation for the one-loop N-point scalar integral. Graphical representation as a sum of $N$ basic dual integrals.

The duality relation between one-loop integrals and phase-space integrals is

$$
L^{(N)}\left(p_{1}, p_{2}, \ldots, p_{N}\right)=-\widetilde{L}^{(N)}\left(p_{1}, p_{2}, \ldots, p_{N}\right),
$$

where the explicit expression of the phase-space integral $\widetilde{L}^{(N)}$ is (Fig. 5)

$$
\widetilde{L}^{(N)}\left(p_{1}, p_{2}, \ldots, p_{N}\right)=\int_{q} \sum_{i=1}^{N} \widetilde{\delta}\left(q_{i}\right) \prod_{\substack{j=1 \\ j \neq i}}^{N} \frac{1}{q_{j}^{2}-i 0 \eta\left(q_{j}-q_{i}\right)},
$$


and $\eta$ is the auxiliary vector in Eq. (31). Each of the $N-1$ propagators in the integrand is regularized by the dual $i 0$ prescription and, thus, it is named 'dual' propagator. Note that the momentum difference $q_{i}-q_{j}$ is independent of the integration momentum $q$ : it only depends on the momenta of the external legs of the loop (see Eq. (2)).

Using the invariance of the integration measure under translations of the momentum $q$, we can perform the same momentum shifts as described in Sect. 3. In analogy to Eq. (23), we can rewrite Eq. (33) as a sum of $N$ basic phase-space integrals (Fig. 5):

$$
\begin{aligned}
\widetilde{L}^{(N)}\left(p_{1}, p_{2}, \ldots, p_{N}\right) & =I^{(N-1)}\left(p_{1}, p_{1}+p_{2}, \ldots, p_{1}+p_{2}+\cdots+p_{N-1}\right)+\text { cyclic perms. } \\
& =\sum_{i=1}^{N} I^{(N-1)}\left(p_{i}, p_{i}+p_{i+1}, \ldots, p_{i}+p_{i+1}+\cdots+p_{i+N-2}\right)
\end{aligned}
$$

The basic one-particle phase-space integrals with $n$ dual propagators are denoted by $I^{(n)}$, and are defined as follows:

$$
I^{(n)}\left(k_{1}, k_{2}, \ldots, k_{n}\right)=\int_{q} \widetilde{\delta}(q) \mathcal{I}^{(n)}\left(q ; k_{1}, k_{2}, \ldots, k_{n}\right)=\int_{q} \widetilde{\delta}(q) \prod_{j=1}^{n} \frac{1}{2 q k_{j}+k_{j}^{2}-i 0 \eta k_{j}} .
$$

We comment on the comparison between the FTT (Eqs. (20)-(24)) and the duality relation (Eqs. (32)-(35)). The multiple-cut contributions $L_{\mathrm{m}-\text { cut }}^{(N)}$, with $m \geq 2$, of the FTT are completely absent from the duality relation. The duality relation involves only singlecut contributions, which are similar to those in $L_{1-\text { cut }}^{(N)}$. On the other hand, the Feynman propagators in $L_{1-\text { cut }}^{(N)}$ are replaced with the dual propagators in $\widetilde{L}^{(N)}$. This replacement in the FTT compensates for the absence of multiple-cut contributions in the duality relation.

The $i 0$ prescription of the dual propagator depends on the auxiliary vector $\eta$. The basic dual integrals $I^{(n)}$ are well defined for arbitrary values of $\eta$. However, when computing $\widetilde{L}^{(N)}$, the future-like vector $\eta$ has to be the same in all its contributing dual integrals (propagators): only then $\widetilde{L}^{(N)}$ does not depend on $\eta$.

In our derivation of the duality relation, the auxiliary vector $\eta$ originates from the use of the residue theorem. Independently of its origin, we can comment on the role of $\eta$ in the duality relation. The one-loop integral $L^{(N)}\left(p_{1}, p_{2}, \ldots, p_{N}\right)$ is a function of the Lorentzinvariants $\left(p_{i} p_{j}\right)$. This function has a complicated analytic structure, with pole and branchcut singularities (scattering singularities), in the multidimensional space of the complex variables $\left(p_{i} p_{j}\right)$. The $i 0$ prescription of the Feynman propagators selects a Riemann sheet in this multidimensional space and, thus, it unambiguously defines $L^{(N)}\left(p_{1}, p_{2}, \ldots, p_{N}\right)$ as a single-valued function. Each single-cut contribution to $\widetilde{L}^{(N)}$ has additional (unphysical) singularities in the multidimensional complex space. The dual $i 0$ prescription fixes the position of these singularities. The auxiliary vector $\eta$ correlates the various single-cut contributions in $\widetilde{L}^{(N)}$, so that they are evaluated on the same Riemann sheet: this leads to the cancellation of the unphysical single-cut singularities. In contrast, in the case of the FTT, this cancellation is produced by the introduction of the multiple-cut contributions $L_{\mathrm{m}-\text { cut }}^{(N)}$.

We remark that the expression $(34)$ of $\widetilde{L}^{(N)}$ as a sum of basic dual integrals is just a matter of notation: for massless internal particles $\widetilde{L}^{(N)}$ is actually a single phase-space 
integral whose integrand is the sum of the terms obtained by cutting each of the internal lines of the loop. In explicit form, we can write:

$$
\widetilde{L}^{(N)}\left(p_{1}, p_{2}, \ldots, p_{N}\right)=\int_{q} \widetilde{\delta}(q) \sum_{i=1}^{N} \mathcal{I}^{(N-1)}\left(q ; p_{i}, p_{i}+p_{i+1}, \ldots, p_{i}+p_{i+1}+\cdots+p_{i+N-2}\right),
$$

where the function $\mathcal{I}^{(n)}$ is the integrand of the dual integral in Eq. (35). Therefore, the duality relation (32) directly expresses the one-loop integral as the phase-space integral of a tree-level quantity. To name Eq. (32), we have introduced the term 'duality' precisely to point out this direct relation* between the $d$-dimensional integral over the loop momentum and the $(d-1)$-dimensional integral over the one-particle phase-space. In the case of the FTT, the relation between loop-level and tree-level quantities is more involved, since the multiple-cut contributions $L_{\mathrm{m}-\text { cut }}^{(N)}$ (with $m \geq 2$ ) contain integrals of expressions that correspond to the product of $m$ tree-level diagrams over the phase-space for a different numbers of particles.

The simpler correspondence between loops and trees in the context of the duality relation is further exploited in Sect. 10, where we discuss Green's functions and scattering amplitudes.

\section{Example: The scalar two-point function}

In this Section we illustrate the application of the FTT and of the duality relation to the evaluation of the one-loop two-point function $L^{(2)}$. A detailed discussion (including detailed results in analytic form and numerical results) of higher-point functions will be presented elsewhere [5] (see also Refs. [3, 4]).

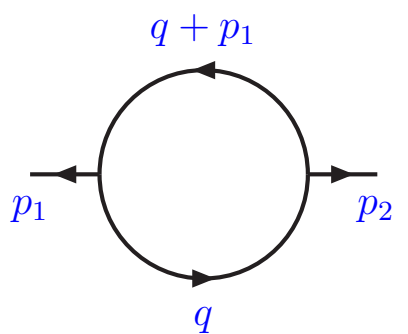

Figure 6: The one-loop two-point scalar integral $L^{(2)}\left(p_{1}, p_{2}\right)$.

The two-point function (Fig. 6), also known as bubble function Bub, is the simplest non-trivial one-loop integral with massless internal lines:

$$
\operatorname{Bub}\left(p_{1}^{2}\right) \equiv L^{(2)}\left(p_{1}, p_{2}\right)=-i \int \frac{d^{d} q}{(2 \pi)^{d}} \frac{1}{\left[q^{2}+i 0\right]\left[\left(q+p_{1}\right)^{2}+i 0\right]} .
$$

Here, we have visibly implemented momentum conservation $\left(p_{1}+p_{2}=0\right)$ and exploited Lorentz invariance $\left(L^{(2)}\left(p_{1}, p_{2}\right)\right.$ can only depend on $p_{1}^{2}$, which is the sole available invariant).

\footnotetext{
*The word duality also suggests a stronger (possibly, one-to-one) correspondence. The correspondence between dual integrals and loop integrals is further discussed in Sect. 7.
} 
Since most of the one-loop calculations have been carried out in four-dimensional field theories (or in their dimensionally-regularized versions), we set $d=4-2 \epsilon$. Note, however, that we present results for arbitrary values of $\epsilon$ or, equivalently, for any value $d$ of space-time dimensions.

The result of the one-loop integral in Eq. (37) is well known:

$$
\operatorname{Bub}\left(p^{2}\right)=c_{\Gamma} \frac{1}{\epsilon(1-2 \epsilon)}\left(-p^{2}-i 0\right)^{-\epsilon},
$$

where $c_{\Gamma}$ is the customary $d$-dimensional volume factor that appears from the calculation of one-loop integrals:

$$
c_{\Gamma} \equiv \frac{\Gamma(1+\epsilon) \Gamma^{2}(1-\epsilon)}{(4 \pi)^{2-\epsilon} \Gamma(1-2 \epsilon)} .
$$

We recall that the $i 0$ prescription in Eq. (38) follows from the corresponding prescription of the Feynman propagators in the integrand of Eq. (37). The $i 0$ prescription defines $\operatorname{Bub}\left(p^{2}\right)$ as a single-value function of the real variable $p^{2}$. In particular, it gives $\operatorname{Bub}\left(p^{2}\right)$ an imaginary part with an unambiguous value when $p^{2}>0$ :

$$
\operatorname{Bub}\left(p^{2}\right)=c_{\Gamma} \frac{1}{\epsilon(1-2 \epsilon)}\left(\left|p^{2}\right|\right)^{-\epsilon}\left[\theta\left(-p^{2}\right)+\theta\left(p^{2}\right) e^{i \pi \epsilon}\right] .
$$

\subsection{General form of single-cut integrals}

To apply the FTT and the duality relation, we have to compute the single-cut integrals $I_{1-\text { cut }}^{(1)}$ and $I^{(1)}$, respectively. Since these integrals only differ because of their $i 0$ prescription, we introduce a more general regularized version, $I_{\text {reg }}^{(1)}$, of the single-cut integral. We define:

$$
I_{\text {reg }}^{(1)}(k ; c(k))=\int_{q} \widetilde{\delta}(q) \frac{1}{2 q k+k^{2}+i 0 c(k)}=\int \frac{d^{d} q}{(2 \pi)^{d-1}} \delta_{+}\left(q^{2}\right) \frac{1}{2 q k+k^{2}+i 0 c(k)} .
$$

Although $c(k)$ is an arbitrary function of $k, I_{\text {reg }}^{(1)}$ only depends on the sign of the $i 0$ prescription, i.e. on the sign of the function $c(k)$ : setting $c(k)=+1$ we recover $I_{1 \text {-cut }}^{(1)}$, cf. Eq. (24), while setting $c(k)=-\eta k$ we recover $I^{(1)}$ (see Eq. (35)).

The calculation of the integral in Eq. (41) is elementary, and the result is

$$
I_{\mathrm{reg}}^{(1)}(k ; c(k))=-\frac{c_{\Gamma}}{2 \cos (\pi \epsilon)} \frac{1}{\epsilon(1-2 \epsilon)}\left[\frac{k^{2}}{k_{0}}-i 0 k^{2} c(k)\right]^{-\epsilon}\left[k_{0}-i 0 k^{2} c(k)\right]^{-\epsilon} .
$$

Note that the typical volume factor, $\widetilde{c}_{\Gamma}$, of the $d$-dimensional phase-space integral is

$$
\widetilde{c}_{\Gamma}=\frac{\Gamma(1-\epsilon) \Gamma(1+2 \epsilon)}{(4 \pi)^{2-\epsilon}} .
$$

The factor $\cos (\pi \epsilon)$ in Eq. (42) originates from the difference between $\widetilde{c}_{\Gamma}$ and the volume factor $c_{\Gamma}$ of the loop integral:

$$
\frac{\widetilde{c}_{\Gamma}}{c_{\Gamma}}=\frac{\Gamma(1+2 \epsilon) \Gamma(1-2 \epsilon)}{\Gamma(1+\epsilon) \Gamma(1-\epsilon)}=\frac{1}{\cos (\pi \epsilon)} .
$$


We also note that the result in Eq. (42) depends on the sign of the energy $k_{0}$. This follows from the fact that the integration measure in Eq. (41) has support on the future light-cone, which is selected by the positive-energy requirement of the on-shell constraint $\delta_{+}\left(q^{2}\right)$.

The denominator contribution $\left(2 q k+k^{2}\right)$ in the integrand of Eq. (41) is positive definite in the kinematical region where $k^{2}>0$ and $k_{0}>0$. In this region the $i 0$ prescription is inconsequential, and $I_{\text {reg }}^{(1)}$ has no imaginary part. Outside this kinematical region, $\left(2 q k+k^{2}\right)$ can vanish, leading to a singularity of the integrand. The singularity is regularized by the $i 0$ prescription, which also produces a non-vanishing imaginary part. The result in Eq. (42) explicitly shows these expected features, since it can be rewritten as

$$
\begin{aligned}
I_{\text {reg }}^{(1)}(k ; c(k)) & =-\frac{c_{\Gamma}}{2 \cos (\pi \epsilon)} \frac{\left(\left|k^{2}\right|\right)^{-\epsilon}}{\epsilon(1-2 \epsilon)}\left\{\theta\left(-k^{2}\right)[\cos (\pi \epsilon)-i \sin (\pi \epsilon) \operatorname{sign}(c(k))]\right. \\
& \left.+\theta\left(k^{2}\right)\left[\theta\left(k_{0}\right)+\theta\left(-k_{0}\right)(\cos (2 \pi \epsilon)+i \sin (2 \pi \epsilon) \operatorname{sign}(c(k)))\right]\right\}
\end{aligned}
$$

We note that the functions $\operatorname{Bub}\left(k^{2}\right)$ and $I_{\text {reg }}^{(1)}(k ; c(k))$ have different analyticity properties in the complex $k^{2}$ plane. The bubble function has a branch-cut singularity along the positive real axis, $k^{2}>0$. The phase-space integral $I_{\text {reg }}^{(1)}(k ; c(k))$ has a branch-cut singularity along the entire real axis if $k_{0}<0$, while the branch-cut singularity is placed along the negative real axis if $k_{0}>0$.

\subsection{Duality relation for the two-point function}

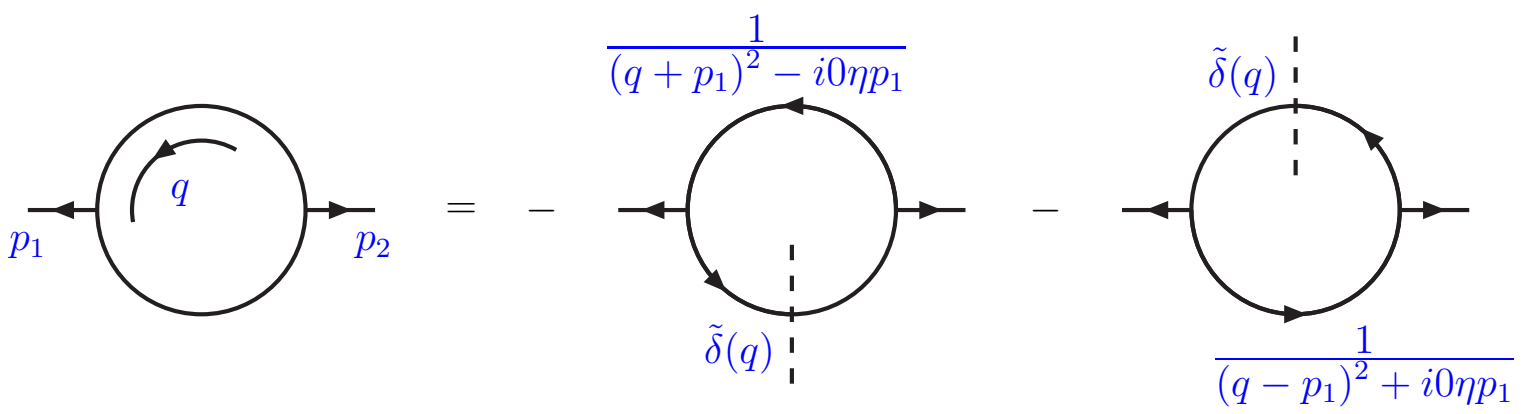

Figure 7: One-loop two-point function: the duality relation.

We now consider the duality relation (Fig. 7) in the context of this example. The dual representation of the one-loop two-point function is given by

$$
\widetilde{L}^{(2)}\left(p_{1}, p_{2}\right)=I^{(1)}\left(p_{1}\right)+\left(p_{1} \leftrightarrow-p_{1}\right),
$$

cf. Eqs. (34) and (35). The basic dual integral $I^{(1)}(k)$ is obtained by setting $c(k)=-\eta k$ in Eq. (42). Since $\eta^{\mu}$ is a future-like vector, $c(k)$ has the following important property:

$$
\operatorname{sign}(\eta k)=\operatorname{sign}\left(k_{0}\right), \quad \text { if } k^{2} \geq 0 .
$$

Using this property, the result in Eq. (42) can be written as

$$
I^{(1)}(k)=-\frac{c_{\Gamma}}{2} \frac{\left(-k^{2}-i 0\right)^{-\epsilon}}{\epsilon(1-2 \epsilon)}\left[1-i \frac{\sin (\pi \epsilon)}{\cos (\pi \epsilon)} \operatorname{sign}\left(k^{2} \eta k\right)\right] .
$$


Comparing this expression with Eq. (38), we see that the imaginary contribution in the square bracket is responsible for the difference with the two-point function. However, since $\operatorname{sign}(-\eta k)=-\operatorname{sign}(\eta k)$, this contribution is odd under the exchange $k \rightarrow-k$ and, therefore, it cancels when Eq. (48) is inserted in Eq. (46). Taken together,

$$
\widetilde{L}^{(2)}\left(p_{1}, p_{2}\right)=I^{(1)}\left(p_{1}\right)+\left(p_{1} \leftrightarrow-p_{1}\right)=-c_{\Gamma} \frac{\left(-p_{1}^{2}-i 0\right)^{-\epsilon}}{\epsilon(1-2 \epsilon)}
$$

which fully agrees with the duality relation $\widetilde{L}^{(2)}\left(p_{1}, p_{2}\right)=-\operatorname{Bub}\left(p_{1}^{2}\right)$.

\subsection{FTT for the two-point function}
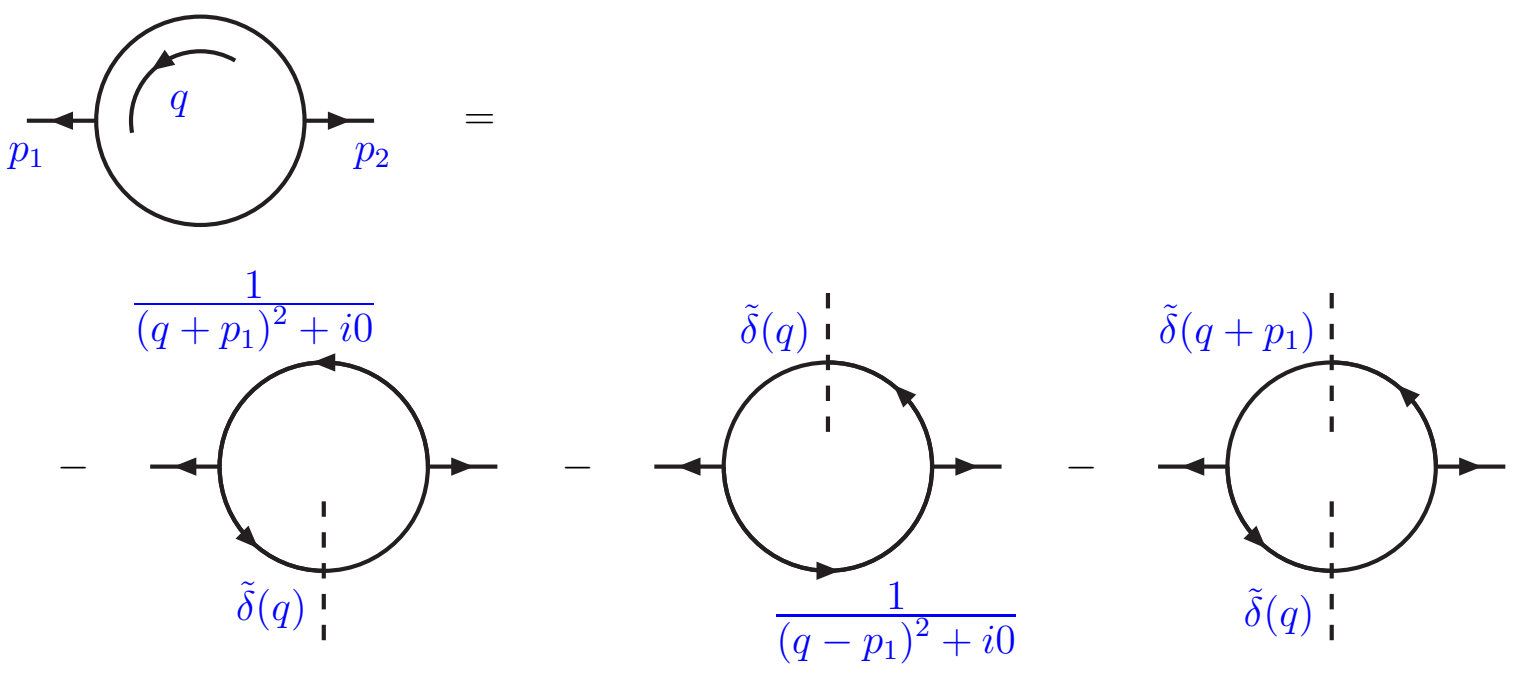

Figure 8: One-loop two-point function: the Feynman Tree Theorem

We now would like to discuss the FTT (Fig. 8) in the case of the two-point function. To this end, we want to check the relations of Eqs. (21)-(24). For the FTT, the two-point function is cast into the form

$$
L^{(2)}\left(p_{1}, p_{2}, \ldots, p_{N}\right)=-\left[L_{1-\text { cut }}^{(2)}\left(p_{1}, p_{2}\right)+L_{2-\text { cut }}^{(2)}\left(p_{1}, p_{2}\right)\right],
$$

where the single-cut and double-cut contributions are

$$
L_{1-\text { cut }}^{(2)}\left(p_{1}, p_{2}\right)=I_{1-\text { cut }}^{(1)}\left(p_{1}\right)+\left(p_{1} \leftrightarrow-p_{1}\right)
$$

and

$$
L_{2-\text { cut }}^{(2)}\left(p_{1}, p_{2}\right)=\int_{q} \widetilde{\delta}(q) \widetilde{\delta}\left(q+p_{1}\right)=i \int \frac{d^{d} q}{(2 \pi)^{d-2}} \theta\left(q_{0}\right) \delta\left(q^{2}\right) \theta\left(q_{0}+p_{10}\right) \delta\left(\left(q+p_{1}\right)^{2}\right)
$$

respectively. The basic single-cut integral $I_{1-\text { cut }}^{(1)}(k)$ of Eq. (51) is obtained by setting $c(k)=+1$ in Eq. (42); we then have

$$
I_{1-\text { cut }}^{(1)}(k)=-\frac{c_{\Gamma}}{2} \frac{\left(-k^{2}-i 0\right)^{-\epsilon}}{\epsilon(1-2 \epsilon)}\left[1-i \frac{\sin (\pi \epsilon)}{\cos (\pi \epsilon)}\left[\theta\left(-k^{2}\right)+\theta\left(k^{2}\right) \operatorname{sign}\left(k_{0}\right)\right]\right] .
$$


Comparing the individual single-cut results, Eqs. (48) and (53), we see that the imaginary contributions in the square brackets are different. Inserting Eq. (53) into Eq. (51), the part of the imaginary contribution that is proportional to $\operatorname{sign}\left(k_{0}\right)$ cancels (this part is odd under the exchange $k \rightarrow-k$ ), while the remaining part does not:

$$
L_{1-\text { cut }}^{(2)}\left(p_{1}, p_{2}\right)=I_{1-\text { cut }}^{(1)}\left(p_{1}\right)+\left(p_{1} \leftrightarrow-p_{1}\right)=-c_{\Gamma} \frac{\left(-p_{1}^{2}-i 0\right)^{-\epsilon}}{\epsilon(1-2 \epsilon)}\left[1-i \frac{\sin (\pi \epsilon)}{\cos (\pi \epsilon)} \theta\left(-p_{1}^{2}\right)\right]
$$

We see that also the sum of the two single-cut contributions of Eqs. (49) and (54) are different: the difference is due to the replacement of the dual $i 0$ prescription with the Feynman $i 0$ prescription. In particular, the difference is a purely imaginary term with support on the space-like region $p_{1}^{2}<0$, whereas the two-point function is purely real in the same region. In the FTT, this difference is compensated by the double-cut contribution $L_{2-\text { cut }}^{(2)}$.

The calculation of the double-cut contribution in Eq. (52) results in

$$
L_{2-\text { cut }}^{(2)}\left(p_{1}, p_{2}\right)=-i c_{\Gamma} \frac{\left(\left|p_{1}^{2}\right|\right)^{-\epsilon}}{\epsilon(1-2 \epsilon)} \frac{\sin (\pi \epsilon)}{\cos (\pi \epsilon)} \theta\left(-p_{1}^{2}\right)
$$

Inserting Eqs. (54) and (55) into the right-hand side of the FTT expression of Eq. (50), we find agreement with the result from the direct one-loop computation of the two-point function.

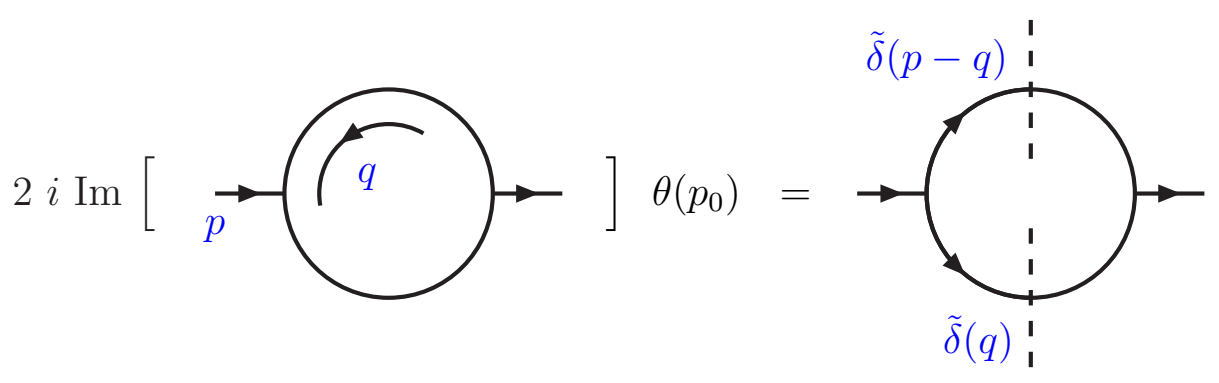

Figure 9: One-loop two-point function: the imaginary part.

To conclude this illustration of the FTT, we add a remark. The double-cut contribution $L_{2-\text { cut }}^{(2)}$ is different from the unitarity-cut contribution that gives the imaginary part of the bubble function (or, equivalently, the discontinuity of $\mathrm{Bub}\left(p^{2}\right)$ across its branch-cut singularity). The imaginary part of the two-point function can be obtained by applying the Cutkosky rules (Fig. 9):

$2 i \operatorname{Im}\left[\operatorname{Bub}\left(p^{2}\right)\right] \theta\left(p_{0}\right)=\int_{q} \widetilde{\delta}(q) \widetilde{\delta}(p-q)=i \int \frac{d^{d} q}{(2 \pi)^{d-2}} \theta\left(q_{0}\right) \delta\left(q^{2}\right) \theta\left(p_{0}-q_{0}\right) \delta\left((q-p)^{2}\right)$.

We see that the double-cut contributions in Eq. (52) and (56) are different due to the determination of the positive-energy flow in the internal lines. Once the energy of the line with momentum $q$ is fixed to be positive, the on-shell line with momentum $q+k$ has positive energy in Eq. (52) and negative energy in Eq. (56). The computation of the double-cut 
integral in Eq. (56) yields

$$
\int_{q} \widetilde{\delta}(q) \widetilde{\delta}(p-q)=+i c_{\Gamma} \frac{\left(\left|p^{2}\right|\right)^{-\epsilon}}{\epsilon(1-2 \epsilon)} 2 \sin (\pi \epsilon) \theta\left(p^{2}\right) \theta\left(p_{0}\right)
$$

which indeed differs from the expression in Eq. (55). Inserting Eq. (57) in Eq. (56), we obtain the imaginary part of $\operatorname{Bub}\left(p^{2}\right)$, in complete agreement with the result (40) of the one-loop integral.

We also note that the Cutkosky rules in Eq. (56) can be derived in a direct way (i.e., without the explicit computation of any integrals) from the duality relation. The derivation is as follows. Applying the identity (18) to the dual propagator, we have

$$
\operatorname{Im}\left[I^{(1)}(p)\right]=\pi \int_{q} \widetilde{\delta}(q) \delta\left((q+p)^{2}\right) \operatorname{sign}(\eta p) .
$$

We now use the duality relation to compute the imaginary part of the two-point function, which is given by

$$
2 i \operatorname{Im}\left[\operatorname{Bub}\left(p^{2}\right)\right] \theta\left(p_{0}\right)=-2 i \theta\left(p_{0}\right)\left[\operatorname{Im} I^{(1)}(p)+(p \leftrightarrow-p)\right] .
$$

Inserting Eq. (58) in Eq. (59), we obtain

$$
\begin{aligned}
& 2 i \operatorname{Im}\left[\operatorname{Bub}\left(p^{2}\right)\right] \theta\left(p_{0}\right)=-2 \pi i \operatorname{sign}(\eta p) \theta\left(p_{0}\right) \int_{q} \widetilde{\delta}(q)\left[\delta\left((q+p)^{2}\right)-\delta\left((q-p)^{2}\right)\right] \\
& =-(2 \pi i)^{2} \operatorname{sign}(\eta p) \theta\left(p_{0}\right) \int_{q} \delta\left(q^{2}\right) \delta\left((q-p)^{2}\right)\left\{\theta\left(q_{0}-p_{0}\right)-\theta\left(q_{0}\right)\right\},
\end{aligned}
$$

where the first term in the square bracket has been rewritten by performing the shift $q \rightarrow q-p$ of the integration variable $q$. The energy constraints in Eq. (60) result in

$$
\theta\left(p_{0}\right)\left\{\theta\left(q_{0}-p_{0}\right)-\theta\left(q_{0}\right)\right\}=-\theta\left(q_{0}\right) \theta\left(p_{0}-q_{0}\right)
$$

This can be inserted in Eq. (60) to obtain

$$
2 i \operatorname{Im}\left[\operatorname{Bub}\left(p^{2}\right)\right] \theta\left(p_{0}\right)=\operatorname{sign}(\eta p) \int_{q} \widetilde{\delta}(q) \widetilde{\delta}(p-q)
$$

We observe that the constraints $q^{2}=(p-q)^{2}=0$ and $q_{0}>0, p_{0}-q_{0}>0$ imply $\operatorname{sign}(\eta q)=$ $\operatorname{sign}(\eta(p-q))=+1$ (see Eq. (47)) and, hence, $\operatorname{sign}(\eta p)=+1$. Therefore Eq. (62) becomes identical to Eq. (56).

\section{Relating Feynman's theorem and the duality theo- rem}

The one-loop integral $L^{(N)}$ can be expressed by using either the FTT or the duality relation. Comparing Eq. (22) with Eq. (32), we thus derive

$$
\widetilde{L}^{(N)}\left(p_{1}, p_{2}, \ldots, p_{N}\right)=L_{1-\text { cut }}^{(N)}\left(p_{1}, p_{2}, \ldots, p_{N}\right)+\cdots+L_{\mathrm{N}-\text { cut }}^{(N)}\left(p_{1}, p_{2}, \ldots, p_{N}\right) .
$$


This expression relates single-cut dual integrals with multiple-cut Feynman integrals. It has been derived in an indirect way, by applying the residue theorem to the evaluation of one-loop integrals.

In this Section we present another proof of Eq. (63). The proof is direct and purely algebraic. It further clarifies the connection between the FTT and the duality relation.

Our starting point is a basic identity between dual and Feynman propagators. The identity applies to the dual propagators when they are inserted in a single-cut integral. Then

$$
\begin{aligned}
\widetilde{\delta}(q) \frac{1}{2 q k+k^{2}-i 0 \eta k} & =\widetilde{\delta}(q)\left[G(q+k)+\theta(\eta k) 2 \pi i \delta\left((q+k)^{2}\right)\right] \\
& =\widetilde{\delta}(q)[G(q+k)+\theta(\eta k) \widetilde{\delta}(q+k)] .
\end{aligned}
$$

The equality on the first line of Eq. (64) directly follows from Eq. (18). The equality on the second line is obtained as follows. Using the constraint $\widetilde{\delta}(q)$, we have $q^{2}=0$ and $q_{0}>0$. Therefore, from Eq. (47) we thus have $\eta q>0$. Using $\eta q>0$ and the constraint $\theta(\eta k)$, we have $\eta(q+k)>0$. Combining $\eta(q+k)>0$ with $(q+k)^{2}=0$, from Eq. (47) we thus have $q_{0}+k_{0}>0$. This enables the replacement $\delta\left((q+k)^{2}\right) \rightarrow \delta_{+}\left((q+k)^{2}\right)$, which finally yields Eq. (64).

\subsection{Two-point function}

The relation (64) can be used to prove Eq. (63). We first consider the case $N=2$. Inserting Eq. (64) in Eq. (35) and comparing with Eqs. (24) and (52), we obtain

$$
I^{(1)}\left(p_{1}\right)=I_{1-\text { cut }}^{(1)}\left(p_{1}\right)+\theta\left(\eta p_{1}\right) \int_{q} \widetilde{\delta}(q) \widetilde{\delta}\left(q+p_{1}\right)=I_{1-\text { cut }}^{(1)}\left(p_{1}\right)+\theta\left(\eta p_{1}\right) L_{2-\text { cut }}^{(2)}\left(p_{1}, p_{2}\right)
$$

We can now use this equation to compute $\widetilde{L}^{(2)}$ :

$$
\widetilde{L}^{(2)}\left(p_{1}, p_{2}\right)=I^{(1)}\left(p_{1}\right)+I^{(1)}\left(p_{2}\right)=L_{1-\text { cut }}^{(2)}\left(p_{1}, p_{2}\right)+\left[\theta\left(\eta p_{1}\right)+\theta\left(\eta p_{2}\right)\right] L_{2-\text { cut }}^{(2)}\left(p_{1}, p_{2}\right) .
$$

This relation is equivalent to Eq. (63), since by merely using momentum conservation, $p_{1}+p_{2}=0$, we find

$$
\theta\left(\eta p_{1}\right)+\theta\left(\eta p_{2}\right)=\theta\left(\eta p_{1}\right)+\theta\left(-\eta p_{1}\right)=1
$$

\subsection{General $N$-point function}

More generally, the identity (64) relates the basic dual integrals $I^{(n)}$ with multiple-cut Feynman integrals. Inserting Eq. (64) in Eq. (35) and using Eq. (24), we obtain

$$
\begin{aligned}
I^{(n)}\left(k_{1}, k_{2}, \ldots, k_{n}\right) & =I_{1-\text { cut }}^{(n)}\left(k_{1}, k_{2}, \ldots, k_{n}\right)+I_{\eta}^{(n)}\left(k_{1}, k_{2}, \ldots, k_{n}\right) \\
& =I_{1-\text { cut }}^{(n)}\left(k_{1}, k_{2}, \ldots, k_{n}\right)+\sum_{m=1}^{n} I_{m, \eta}^{(n)}\left(k_{1}, k_{2}, \ldots, k_{n}\right),
\end{aligned}
$$


where

$$
\begin{aligned}
I_{m, \eta}^{(n)}\left(k_{1}, k_{2}, \ldots, k_{n}\right)=\int_{q} \widetilde{\delta}(q) & \left\{\widetilde{\delta}\left(q+k_{1}\right) \ldots \widetilde{\delta}\left(q+k_{m}\right) G\left(q+k_{m+1}\right) \ldots G\left(q+k_{n}\right)\right. \\
& \left.\times \theta\left(\eta k_{1}\right) \ldots \theta\left(\eta k_{m}\right)+\text { uneq. perms. }\right\} .
\end{aligned}
$$

Note that the key difference between $I_{m, \eta}^{(n)}$ and the multiple-cut contributions to the FTT (see Eq. (21)) is the presence of the momentum constraints, $\theta\left(\eta k_{i}\right)$, in Eq. (69).

For a proof in the case of the $N$-point function, we employ the following relation:

$$
I_{m-1, \eta}^{(N-1)}\left(p_{1}, p_{1}+p_{2}, \ldots, p_{1}+p_{2}+\cdots+p_{N-1}\right)+\text { cyclic perms. }=L_{\mathrm{m}-\mathrm{cut}}^{(N)}\left(p_{1}, p_{2}, \ldots, p_{N}\right) .
$$

Summing over the cyclic permutations of $I^{(N-1)}$ as in Eq. (34), and using Eqs. (68), (23) and (70), we straightforwardly obtain the relation in Eq. (63).

We note that the proof of Eq. (70) is mainly a matter of combinatorics, and it does not require the explicit evaluation of any $m$-cut integrals. Eventually, the only key ingredient of the proof is the following algebraic identity

$$
\theta\left(\eta p_{1}\right) \theta\left(\eta\left(p_{1}+p_{2}\right)\right) \ldots \theta\left(\eta\left(p_{1}+p_{2}+\cdots+p_{N-1}\right)\right)+\text { cyclic perms. }=1 .
$$

It is a direct consequence of momentum conservation, namely $\sum_{i=1}^{N} p_{i}=0$. The derivation of Eq. (71) is presented in Appendix B.

To simplify the combinatorics in the proof of Eq. (70), we first rewrite $I_{m, \eta}^{(n)}$ in Eq. (69) as

$$
I_{m, \eta}^{(n)}\left(k_{1}, k_{2}, \ldots, k_{n}\right)=I_{m, F}^{(n)}\left(k_{1}, k_{2}, \ldots, k_{n}\right)+\delta I_{m, \eta}^{(n)}\left(k_{1}, k_{2}, \ldots, k_{n}\right),
$$

where

$$
\begin{aligned}
I_{m, F}^{(n)}\left(k_{1}, k_{2}, \ldots, k_{n}\right)=\frac{1}{m+1} \int_{q} \widetilde{\delta}(q) & \left\{\widetilde{\delta}\left(q+k_{1}\right) \ldots \widetilde{\delta}\left(q+k_{m}\right) G\left(q+k_{m+1}\right) \ldots G\left(q+k_{n}\right)\right. \\
& + \text { uneq. perms. }\}
\end{aligned}
$$

and

$$
\begin{aligned}
\delta I_{m, \eta}^{(n)}\left(k_{1}, k_{2}, \ldots, k_{n}\right)=\int_{q} \widetilde{\delta}(q) & \left\{\widetilde{\delta}\left(q+k_{1}\right) \ldots \widetilde{\delta}\left(q+k_{m}\right) G\left(q+k_{m+1}\right) \ldots G\left(q+k_{n}\right)\right. \\
& \left.\times\left[\theta\left(\eta k_{1}\right) \ldots \theta\left(\eta k_{m}\right)-\frac{1}{m+1}\right]+\text { uneq. perms. }\right\} .
\end{aligned}
$$

This leaves us with the task to prove the relations

$$
I_{m-1, F}^{(N-1)}\left(p_{1}, p_{1}+p_{2}, \ldots, p_{1}+p_{2}+\cdots+p_{N-1}\right)+\text { cyclic perms. }=L_{\mathrm{m}-\mathrm{cut}}^{(N)}\left(p_{1}, p_{2}, \ldots, p_{N}\right)
$$

and

$$
\delta I_{m-1, \eta}^{(N-1)}\left(p_{1}, p_{1}+p_{2}, \ldots, p_{1}+p_{2}+\cdots+p_{N-1}\right)+\text { cyclic perms. }=0 .
$$

Obviously, Eqs. (72), (75) and (76) imply Eq. (70). 
The relation (75) can be proven as follows. According to Eq. $(21), L_{\mathrm{m}-\text { cut }}^{(N)}$ is a sum of $m$-cut contributions with a fully symmetric dependence on the momenta $q_{i}$ of the internal lines of the loop integral. The expression on the left-hand side of Eq. (75) is also a fullysymmetric linear combination of $m$-cut contributions: the symmetrization follows from the sum over the permutations in Eqs. (73) and (75). Owing to their symmetry, the lefthand side and the right-hand side of Eq. (75) are necessarily proportional. Actually, the proportionality coefficient is just unity. To show this, we can give weight unity to each $m$ cut contribution and, then, we can simply count the number of $m$-cut contributions on both sides of Eq. (75). The number of terms in $L_{\mathrm{m}-\text { cut }}^{(N)}$ equals the total number of permutations in the curly bracket of Eq. (21), namely

$$
\left(\begin{array}{l}
N \\
m
\end{array}\right)=\frac{N !}{m !(N-m) !} .
$$

The number of terms in the left-hand side of Eq. (75) is

$$
\frac{1}{m}\left(\begin{array}{c}
N-1 \\
m-1
\end{array}\right) N=\frac{1}{m} \frac{(N-1) !}{(m-1) !(N-m) !} N
$$

where, on the left-hand side, the factor $1 / m$ is the weight of each contribution to $I_{m-1, F}^{(N-1)}$, the factor $\left(\begin{array}{c}N-1 \\ m-1\end{array}\right)$ is the number of permutations that contribute to $I_{m-1, F}^{(N-1)}$ (see Eq. (73)), and the factor $N$ is the number of cyclic permutations in Eq. (75). As we can see, the numbers in Eqs. (77) and (78) coincide, thus yielding the equality in Eq. (75).

The relation (76) can be proven as follows. The left-hand side is a sum of $m$-cut contributions of the loop integral $L^{(N)}$. We can organize these contributions in a sum of $\left(\begin{array}{l}N \\ m\end{array}\right)$ diagrams as in the right-hand side of Eq. (21): each diagram has $m$ fixed internal lines that have been cut. The coefficient of each diagram is computed according to the expression on the left-hand side of Eq. (76). As discussed below, this coefficient vanishes algebraically, thus yielding the result in Eq. (76).

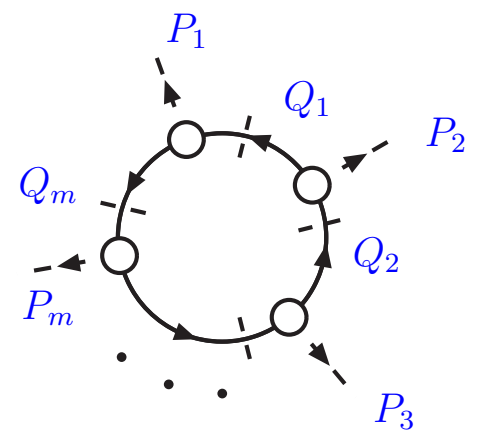

Figure 10: A one-loop diagram with $m$ cut lines. Each blob denotes a set of internal lines that are not cut.

We consider one of the diagram with $m$ cut lines, and we denote the momenta of these internal lines as $Q_{1}, Q_{2}, \ldots, Q_{m}$ (Fig. 10). We define $P_{i}=Q_{i}-Q_{i-1}$, so that $P_{i}$ is the total momentum of the external lines joined by the Feynman propagators inserted between the cut lines with momenta $Q_{i}$ and $Q_{i-1}$. The computation of the diagram involves the factor

$$
\widetilde{\delta}\left(Q_{1}\right) \widetilde{\delta}\left(Q_{2}\right) \ldots \widetilde{\delta}\left(Q_{m}\right)
$$


and two other factors. One factor is due to the product of the Feynman propagators of those internal lines that are not cut; this factor is inconsequential to the present discussion. The other factor is due to the term in the square bracket on the right-hand side of Eq. (74). We note that $\delta I_{m-1, \eta}^{(N-1)}$ involves the product $\widetilde{\delta}(q) \widetilde{\delta}\left(q+k_{1}\right) \ldots \widetilde{\delta}\left(q+k_{m-1}\right)$ of $m$ delta functions, but the term in the square bracket is symmetric only with respect to the argument of $m-1$ delta functions. Therefore, inserting Eq. (74) into Eq. (76) and performing the sum over the permutations, the term in the square bracket leads to $m$ different contributions: each contribution corresponds to one of the assignments $\widetilde{\delta}(q) \rightarrow \widetilde{\delta}\left(Q_{i}\right)$ with $i=1,2, \ldots, m$. In conclusion, the square bracket term contributes to multiply the left-hand side of Eq. (79) by a factor that is proportional to the following expression:

$$
\begin{aligned}
& {\left[\theta\left(\eta P_{1}\right) \theta\left(\eta\left(P_{1}+P_{2}\right)\right) \ldots \theta\left(\eta\left(P_{1}+P_{2}+\cdots+P_{m-1}\right)\right)-\frac{1}{m}\right]+\text { cyclic perms. }} \\
& =\left\{\theta\left(\eta P_{1}\right) \theta\left(\eta\left(P_{1}+P_{2}\right)\right) \ldots \theta\left(\eta\left(P_{1}+P_{2}+\cdots+P_{m-1}\right)\right)+\text { cyclic perms. }\right\}-1 .
\end{aligned}
$$

This expression actually vanishes, because of Eq. (71) and the momentum conservation constraint $\sum_{i=1}^{m} P_{i}=0$. Therefore, each of the $m$-cut diagrams that contributes to the left-hand side of Eq. (76) has a vanishing coefficient.

\section{$7 \quad$ Dual bases and generalized duality}

One-loop Feynman integrals and single-cut dual integrals are not in a one-to-one correspondence. Starting from this observation we discuss in more general terms the nature of the correspondence between one-loop and single-cut integrals in this section.

Using the duality relation, any one-loop Feynman integral $L^{(N)}$ can be expressed as a linear combination of the basic dual integrals $I^{(N-1)}$, but the opposite statement is not true. Therefore, the dual integrals $I^{(n)}$ form a linear basis of the functional space generated by the loop integrals, but in total they generate a space that is larger than the space of the one-loop Feynman integrals.

To express $I^{(N-1)}$ as a linear combination of loop integrals, we have to introduce generalized one-loop integrals, whose integrand contains both Feynman and advanced propagators. We define them through

$$
L^{(N)}\left(p_{1}, \alpha_{1}, p_{2}, \alpha_{2}, \ldots, p_{N}, \alpha_{N}\right)=\int_{q} \prod_{i=1}^{N} G_{\alpha_{i}}\left(q_{i}\right)
$$

where the label $\alpha_{i}$ can take two values, $\alpha_{i}=F, A$, and $G_{F}\left(q_{i}\right)=G\left(q_{i}\right)$ is the Feynman propagator, while $G_{A}\left(q_{i}\right)$ is the advanced propagator. In particular, when $\alpha_{1}=\alpha_{2}=\cdots=$ $\alpha_{N}=F$ we recover the one-loop Feynman integral in Eq. (9), while we obtain the one-loop advanced integral in Eqs. (14) and (15) for the case $\alpha_{1}=\alpha_{2}=\cdots=\alpha_{N}=A$.

The relation between $I^{(N-1)}$ and the generalized one-loop integrals in Eq. (81) is obtained by noticing that the dual propagator can be rewritten as a linear combination of $G$ 
and $G_{A}$. Using Eqs. (17) and (64) we have:

$$
\begin{aligned}
\widetilde{\delta}(q) \frac{1}{2 q k+k^{2}-i 0 \eta k} & =\widetilde{\delta}(q)\left[G(q+k)+\theta(\eta k)\left(G_{A}(q+k)-G(q+k)\right)\right] \\
& =\widetilde{\delta}(q)\left[\theta(-\eta k) G(q+k)+\theta(\eta k) G_{A}(q+k)\right],
\end{aligned}
$$

which can be inserted in Eq. (35). We thus obtain

$$
\begin{aligned}
I^{(n)}\left(k_{1}, k_{2}, \ldots, k_{n}\right) & =\int_{q} \widetilde{\delta}(q) \prod_{j=1}^{n}\left[\theta\left(-\eta k_{j}\right) G\left(q+k_{j}\right)+\theta\left(\eta k_{j}\right) G_{A}\left(q+k_{j}\right)\right] \\
& =\int_{q}\left(G_{A}(q)-G(q)\right) \prod_{j=1}^{n}\left[\theta\left(-\eta k_{j}\right) G\left(q+k_{j}\right)+\theta\left(\eta k_{j}\right) G_{A}\left(q+k_{j}\right)\right],
\end{aligned}
$$

where again we have used Eq. (17) to express $\widetilde{\delta}(q)$ as a linear combination of $G(q)$ and $G_{A}(q)$. The right-hand side of Eq. (83) is a sum of generalized one-loop integrals. Note that the $\eta$ dependence of $I^{(n)}$ appears only in the coefficients $\theta\left( \pm \eta k_{j}\right)$ that multiply the one-loop integrals.

In the simplest case, with $n=1$, Eq. (83) reads:

$$
\begin{aligned}
I^{(1)}\left(p_{1}\right) & =-\theta\left(-\eta p_{1}\right) \int_{q} G(q) G\left(q+p_{1}\right) \\
& +\left[\theta\left(-\eta p_{1}\right) \int_{q} G_{A}(q) G\left(q+p_{1}\right)-\theta\left(\eta p_{1}\right) \int_{q} G(q) G_{A}\left(q+p_{1}\right)\right] \\
& =-\theta\left(-\eta p_{1}\right) L^{(2)}\left(p_{1},-p_{1}\right)+\left[\theta\left(-\eta p_{1}\right) L^{(2)}\left(p_{1}, F,-p_{1}, A\right)-\left(p_{1} \leftrightarrow-p_{1}\right)\right],
\end{aligned}
$$

where we have used Eq. (15). Note that the term in the square bracket is odd under the exchange $p_{1} \leftrightarrow-p_{1}$. Therefore the sum $I^{(1)}\left(p_{1}\right)+I^{(1)}\left(-p_{1}\right)$ consistently reproduces the duality relation (i.e., equivalently, it reproduces the two-point function $L^{(2)}\left(p_{1},-p_{1}\right)$ ).

More generally, the linear relation in Eq. (83) implies that the dual integrals $I^{(N-1)}$ belong to the functional space that is generated by the generalized one-loop integrals of Eq. (81)

Nonetheless, we are not yet to the point of establishing a one-to-one correspondence between single-cut and one-loop integrals. In fact, the correspondence in Eq. (83) is not invertible. The generalized one-loop integrals can be expressed in terms of single-cut integrals by a proper generalization of the duality relation in Eqs. (32) and (33). However, the single-cut integrals of this generalized relation involve the integration of both dual and advanced propagators.

The generalized duality relation is:

$$
\begin{aligned}
L^{(N)}\left(p_{1}, \alpha_{1}, p_{2}, \alpha_{2}, \ldots, p_{N}, \alpha_{N}\right) & =-\int_{q} \sum_{i=1}^{N} \widetilde{\delta}\left(q_{i}\right) \delta_{\alpha_{i}, F} \\
& \times \prod_{\substack{j=1 \\
j \neq i}}^{N}\left[\delta_{\alpha_{j}, F} \frac{1}{q_{j}^{2}-i 0 \eta\left(q_{j}-q_{i}\right)}+\delta_{\alpha_{j}, A} G_{A}\left(q_{j}\right)\right]
\end{aligned}
$$


This result can be derived by applying the residue theorem (see Appendix A).

Alternatively, Eq. (85) can also be derived by applying an algebraic procedure that is similar to the procedure used in Sect. 6 to prove Eq. (63). This procedure consists in rewriting the right-hand side of Eqs. (81) and (85) as multiple-cut integrals of expressions involving only advanced propagators. Then these expressions can be directly compared to show that they agree with each other. The rewrite of Eqs. (81) and (85) is achieved by using Eq. (17) to replace Feynman and dual propagators with advanced propagators. More precisely, in the case of the dual propagators, Eqs. (17) and (82) give:

$$
\widetilde{\delta}(q) \frac{1}{2 q k+k^{2}-i 0 \eta k}=\widetilde{\delta}(q)\left[G_{A}(q+k)-\theta(-\eta k) \widetilde{\delta}(q+k)\right] .
$$

To exemplify this algebraic procedure, we can explicitly show its application to the simple, though non-trivial, case of the one-loop integral $L^{(3)}\left(p_{1}, F, p_{2}, F, p_{3}, A\right)$. The righthand side of Eq. (81) yields

$$
\begin{aligned}
& \int_{q} G_{A}(q) G\left(q+p_{1}\right) G\left(q+p_{1}+p_{2}\right)=-\int_{q} G_{A}(q) \\
& \times\left[\widetilde{\delta}\left(q+p_{1}\right) G_{A}\left(q+p_{1}+p_{2}\right)+\widetilde{\delta}\left(q+p_{1}+p_{2}\right) G_{A}\left(q+p_{1}\right)-\widetilde{\delta}\left(q+p_{1}\right) \widetilde{\delta}\left(q+p_{1}+p_{2}\right)\right],
\end{aligned}
$$

where we have also used Eq. (15). After using Eq. (86), the right-hand side of Eq. (85) reads

$$
\begin{gathered}
-\int_{q} G_{A}(q)\left[\widetilde{\delta}\left(q+p_{1}\right) \frac{1}{\left(q+p_{1}+p_{2}\right)^{2}-i 0 \eta p_{2}}+\widetilde{\delta}\left(q+p_{1}+p_{2}\right) \frac{1}{\left(q+p_{1}\right)^{2}+i 0 \eta p_{2}}\right] \\
=-\int_{q} G_{A}(q)\left[\widetilde{\delta}\left(q+p_{1}\right)\left(G_{A}\left(q+p_{1}+p_{2}\right)-\theta\left(-\eta p_{2}\right) \widetilde{\delta}\left(q+p_{1}+p_{2}\right)\right)\right. \\
\left.+\widetilde{\delta}\left(q+p_{1}+p_{2}\right)\left(G_{A}\left(q+p_{1}\right)-\theta\left(\eta p_{2}\right) \widetilde{\delta}\left(q+p_{1}\right)\right)\right] .
\end{gathered}
$$

By simple inspection, we see that the expressions in Eqs. (87) and (88) coincide.

The generalized duality in Eq. (85) relates one-loop integrals to single-cut phase-space integrals. Note that only the Feynman propagators of the loop integral are cut; the Feynman propagators that are not cut are instead replaced by dual propagators. The advanced propagators of the loop integral are not cut, and they appear unchanged in the integrand of the phase-space integral.

The correspondence in Eq. (85) between one-loop and single-cut integrals is invertible. Using the same algebraic steps as in Eqs. (82) and (83), we indeed obtain:

$$
\begin{aligned}
& \int_{q} \widetilde{\delta}(q)\left(\prod_{j=1}^{m} \frac{1}{2 q k_{j}+k_{j}^{2}-i 0 \eta k_{j}}\right) \prod_{i=1}^{k} G_{A}\left(q+k_{i}\right) \\
& =\int_{q}\left(G_{A}(q)-G(q)\right) \prod_{j=1}^{m}\left[\theta\left(-\eta k_{j}\right) G\left(q+k_{j}\right)+\theta\left(\eta k_{j}\right) G_{A}\left(q+k_{j}\right)\right] \prod_{i=1}^{k} G_{A}\left(q+k_{i}\right) .
\end{aligned}
$$

The functional space generated by the generalized one-loop integrals is thus equivalent to the space generated by the single-cut integrals on the left-hand side of Eq. (89). The oneloop integrals of Feynman and advanced propagators and the single-cut integrals of dual and advanced propagators can be regarded as equivalent dual basis of this functional space. 


\section{Massive integrals, complex masses and unstable particles}

As discussed at the end of Sect. 3, the introduction of particle masses and massive propagators does not lead to any difficulties in the generalization of the FTT from the massless case. The same discussion and the same conclusions apply to the duality relation, since this relation can be derived by applying the residue theorem in close analogy with the derivation of the FTT. Therefore, as long as the mass is real, the effect of a particle mass $M_{i}$ in the Feynman propagator of a loop internal line with momentum $q_{i}$ amounts to modifying (according to the replacement in Eq. (26)) the corresponding on-shell delta function $\widetilde{\delta}\left(q_{i}\right)$ when this line is cut to obtain the dual representation $\widetilde{L}^{(N)}$ (see Eqs. (33) and (85)) of the loop integral $L^{(N)}$. Note also that the $i 0$ prescription of the dual propagators is not affected by the masses. More precisely, if the Feynman propagator of the $j$-th internal line has mass $M_{j}$, the corresponding dual propagator is

$$
\frac{1}{q_{j}^{2}-M_{j}^{2}-i 0 \eta\left(q_{j}-q_{i}\right)}
$$

independently of the value $M_{i}$ of the mass in the $i$-th line (the cut line).

In any unitary quantum field theory, the masses of the basic fields are real. If some of these fields describe unstable particles, a proper (physical) treatment of the corresponding propagators in perturbation theory requires a Dyson summation of self-energy insertions. The Dyson summation produces finite-width effects that lead to the introduction of finite imaginary contributions in the propagators. A typical form of the ensuing propagator $G_{C}$ (such as the propagator used in the complex-mass scheme ${ }^{\dagger}[10]$ ) is

$$
G_{C}(q ; s)=\frac{1}{q^{2}-s}
$$

where $s$ denotes the complex mass of the unstable particle:

$$
s=\operatorname{Re} s+i \operatorname{Im} s, \quad \text { with } \quad \operatorname{Re} s>0>\operatorname{Im} s .
$$

This complex mass, together with complex couplings, is introduced in both tree- and oneloop Feynman diagrams. A natural question that arises in the context of the present paper is whether the duality relation between one-loop and phase-space integrals (and the FTT, as well) can deal with complex-mass propagators or, more generally, with propagators of unstable particles. The answer to this question is positive, as we discuss below.

We consider a one-loop $N$-point scalar integral (see Eq. (9)) where one or more of the Feynman propagators of the internal lines are replaced by complex-mass propagators $G_{C}\left(q_{i} ; s_{i}\right)$. To derive a representation of this one-loop integral in terms of single-cut phase space integral, we then apply the same procedure as in Sect. 4. The only difference with respect to the discussion in Sect. 4 is due to the presence of the complex-mass propagators. In the complex plane of the loop integration variable $q_{0}$, the complex-mass propagators

\footnotetext{
${ }^{\dagger}$ In the complex-mass scheme, unitarity can be perturbatively recovered (modulo higher-order terms) order by order.
} 
produce poles that are located far from the real axis, the displacement from the real axis being controlled by the finite imaginary part of the complex masses. Using the Cauchy theorem as in Eq. (27), we derive a duality relation that is analogous to Eq. (32). The only difference is that the the right-hand side of Eq. (32) has to be modified:

$$
\widetilde{L}^{(N)}\left(p_{1}, p_{2}, \ldots, p_{N}\right) \rightarrow \widetilde{L}^{(N)}\left(p_{1}, p_{2}, \ldots, p_{N}\right)+\widetilde{L}_{C}^{(N)}\left(p_{1}, p_{2}, \ldots, p_{N}\right)
$$

Here, $\widetilde{L}^{(N)}$ denotes the terms that correspond to the residues at the poles of the Feynman propagators of the loop integral, while $\widetilde{L}_{C}^{(N)}$ denotes the terms from the poles of the complexmass propagators.

$\widetilde{L}^{(N)}$ is thus expressed as

$$
\widetilde{L}^{(N)}\left(p_{1}, p_{2}, \ldots, p_{N}\right)=\int_{q} \sum_{i \in F} \widetilde{\delta}\left(q_{i} ; M_{i}\right)\left[\prod_{j \neq i} \ldots\right],
$$

where the sum refers to the internal lines $i$ of the loop with a Feynman propagator (we use the notation $i \in F$ to denote these cut lines). The term in the square bracket denotes the product of the propagators of the lines that are not cut. The Feynman propagators of the loop are replaced by the corresponding dual propagators (as in Eq. (33)), while the complex-mass propagators are unchanged ${ }^{\ddagger}$.

The expression of $\widetilde{L}_{C}^{(N)}$ is similar to Eq. (94), but the cut lines $i$ are those with complexmass propagators (we use the notation $i \in C$ to denote these cut lines). Taken together

$$
\begin{aligned}
\widetilde{L}_{C}^{(N)}\left(p_{1}, p_{2}, \ldots, p_{N}\right) & =\int_{q} \sum_{i \in C} \widetilde{\delta}\left(q_{i} ; s_{i}\right)\left[\prod_{j \neq i} \cdots\right] \\
& =\int \frac{d^{d-1} \mathbf{q}}{(2 \pi)^{d-1}} \sum_{i \in C} \frac{1}{2 \sqrt{\mathbf{q}_{i}^{2}+s_{i}}}\left[\prod_{j \neq i} \ldots\right]_{q_{i 0}=\sqrt{\mathbf{q}_{i}^{2}+s_{i}}},
\end{aligned}
$$

where the term in the square bracket contains the propagators of those lines that are not cut. Note that in the integral representation on the first line of Eq. (95) the 'on-shell' delta function $\widetilde{\delta}\left(q_{i} ; s_{i}\right)$ of the cut propagator has a formal meaning, since it singles out the residue at the complex-mass pole, $q_{i 0}=q_{i 0}^{(C,+)}=\sqrt{\mathbf{q}_{i}^{2}+s_{i}}$, which has a finite (and negative) imaginary part. The explicit expression of $\widetilde{L}_{C}^{(N)}$ is thus given in the second line of Eq. (95). We also note that, owing to the finite imaginary component of $q_{i 0}^{(C,+)}$, we can remove the $i 0$ prescription in any of the Feynman propagators inside the square bracket.

To summarize: the outcome of our discussion of the duality relation can also be used to explain how the FTT can be generalized to deal with complex-mass propagators in the internal lines. Following the derivation of the FTT in Sect. 3, we can replace the advanced one-loop integral $L_{A}^{(N)}$ of Eq. (14) with a one-loop integral that contains both advanced propagators and complex-mass propagators. This one-loop integral can then be rewritten

\footnotetext{
$\ddagger$ The dual propagators arise from the infinitesimal $i 0$ displacement that is produced by the residue at the pole of the Feynman propagator, see Sect. 4 and Appendix A. This infinitesimal imaginary displacement has no effect on the complex-mass propagators, because of the finite imaginary part of the complex mass.
} 
in two different ways. Using one way (i.e. exploiting the relation in Eq. (17)), it can be expressed, as in the right-hand side of Eq. (19), in terms of a linear combination of the required one-loop integral (i.e. the integral with Feynman and complex-mass propagators) and of multiple-cut phase-space integrals $L_{\mathrm{m}-\text { cut }}^{(N)}$. Using the alternative way, it can be evaluated directly by applying the Cauchy theorem as in Eq. (16). This direct evaluation leads to the computation of the residues at the poles of the complex-mass propagators (the poles of the advanced propagators do not contribute, since they are placed outside the integration contour): the computation gives exactly the contribution in Eq. (95). Comparing the expressions obtained in these two ways, we conclude that the generalization of the FTT to include complex-mass propagators is realized by the following replacement in the right-hand side of Eq. (22):

$$
L_{1-\text { cut }}^{(N)}\left(p_{1}, p_{2}, \ldots, p_{N}\right) \rightarrow L_{1-\text { cut }}^{(N)}\left(p_{1}, p_{2}, \ldots, p_{N}\right)+\widetilde{L}_{C}^{(N)}\left(p_{1}, p_{2}, \ldots, p_{N}\right) .
$$

Here, $L_{1-\text { cut }}^{(N)}$ is the usual contribution (see Eq. (20)) from the single cuts of the sole Feynman propagators (the complex-mass propagators are not cut) of the internal lines, while $\widetilde{L}_{C}^{(N)}$ is given by Eq. (95). Note, in particular, that the complex-mass propagators do not produce any additional (i.e., in addition to the real-mass terms $L_{\mathrm{m}-\text { cut }}^{(N)}$ in Eq. (21)) $m$-cut contributions $(m \geq 2)$ to the FTT.

We can add a final comment on the one-loop integrals with unstable particles in the internal lines. The propagator of an unstable particle can have a form that differs from the complex-mass propagator in Eq. (91). We can introduce, for instance, a complex mass, $s\left(q^{2}\right)$, that depends on the momentum $q$ of the propagator. We can also include a non-resonant component, in addition to the resonant contribution of the complex-mass pole. Independently of its specific form, the propagator of the unstable particle produces singularities that are located at a finite imaginary distance from the real axis in the complex plane of the loop integration variable $q_{0}$. Contributions of these unstable particles can be included in the duality relation and in the FTT by performing the replacements in Eq. (93) and in Eq. (96), respectively. In general, the term $\widetilde{L}_{C}^{(N)}$ has a form that differs from Eq. (95) and depends on the actual expression of the propagator and in particular, on the singularity structure (poles, branch cuts, ... ) of the propagator in the complex plane.

\section{Gauge theories and gauge poles}

The quantization of gauge theories requires the introduction of a gauge-fixing procedure. This procedure specifies the spin polarization vectors of the gauge bosons and the ensuing content of (possible) compensating fictitious particles (e.g. the Faddeev-Popov ghosts in unbroken non-Abelian gauge theories, or the would-be Goldstone bosons in spontaneouslybroken gauge theories).

The fictitious particles have their own Feynman propagators, which have to be taken into account when applying either the FTT or the duality relation. This is done in a straightforward manner: if some internal lines in a one-loop integral correspond to fictitious particles, they have to be cut exactly in the same way as for physical particles. The multiplecut phase-space integrals of the FTT and the single-cut phase-space integral of the duality 
relation will include the contributions from the cuts of the Feynman propagators of these fictitious particles.

The impact of the propagators of the gauge particles is more delicate, since they introduce 'gauge poles'. This point is discussed below.

The propagator of the (spin 1) gauge boson with momentum $q$ is obtained by multiplying the customary Feynman propagator $G(q)$ with the tensor $d^{\mu \nu}(q)$, which arises from the sum of the spin polarizations. The general form of the polarization tensor is

$$
d^{\mu \nu}(q)=-g^{\mu \nu}+(\zeta-1) \ell^{\mu \nu}(q) G_{G}(q) .
$$

The second term on the right-hand side is absent only in the 't Hooft-Feynman gauge $(\zeta=$ $1)$. In any other gauge, this term is present and the tensor $\ell^{\mu \nu}(q)$ propagates longitudinal polarizations, which are proportional to $q^{\mu}$, or $q^{\nu}$, or $q^{\mu} q^{\nu}$. The specific form of $\ell^{\mu \nu}(q)$ is not relevant in the context of the following discussion; the only relevant point is that $\ell^{\mu \nu}(q)$ has a polynomial dependence on the momentum $q$. On the other hand, the factor $G_{G}(q)$ (we call it 'gauge-mode' propagator) has a potentially dangerous, non-polynomial dependence on $q$ and, in particular, it produces poles with respect to the momentum variable $q$.

When considering one-loop quantities in gauge theories, we deal with one-loop integrals with gauge boson propagators in the internal lines of the loop. Therefore, to derive the FTT or the duality relation, we have to consider the effect produced by the gauge polarization tensors. In the 't Hooft-Feynman gauge the effect is harmless: the polarization tensor is simply $-g^{\mu \nu}$ and it factorizes from the loop integration. When applying the Cauchy residue theorem as in Sects. 3 and 4 in any other gauge, we have to take into account the possible additional contributions that arise from the presence of the poles of the gaugemode propagator $G_{G}(q)$ (the presence of polynomial terms from $\ell^{\mu \nu}(q)$ does not interfere with the residue theorem).

We first discuss the case of spontaneously-broken gauge theories. Here, the gauge boson has a finite mass $M$, and the form of the gauge-mode propagator $G_{G}(q)$ is

$$
G_{G}(q)=\frac{1}{\zeta\left(q^{2}+i 0\right)-M^{2}}
$$

Considering the unitary gauge $(\zeta=0)$, the gauge-mode propagator does not depend on $q$ and factorizes from the loop integration in any one-loop integrals. Therefore, the unitary gauge has only inconsequential implications on the use of the FTT and the duality relation for one-loop calculations in gauge theories. If we instead consider a generic renormalizable gauge (or $R_{\zeta}$ gauge) with $\zeta \neq 0$, we see that the gauge-mode propagator introduces a pole when $q^{2}=M^{2} / \zeta-i 0$. This is an additional pole with respect to the physical pole (when $\left.q^{2}=M^{2}-i 0\right)$ from the associated Feynman propagators. If one is interested in extending the FTT and the duality relation of Sects. 3 and 4 for actual one-loop computations in the $R_{\zeta}$ gauge, one has to properly consider the introduction of additional single-cut and multiple-cut contributions from gauge-mode propagators. We will not pursue this issue any further in the present paper.

We now discuss the case of unbroken gauge theories, where the gauge boson is massless. We separately consider two classes of gauges: covariant gauges and physical gauges. 
In covariant gauges, we have

$$
G_{G}(q)=\frac{1}{q^{2}+i 0}
$$

Since the gauge-mode propagator $G_{G}(q)$ is equal to the Feynman propagator, the two propagators combine their effect to produce a second-order pole when $q^{2}=-i 0$. The extension of the FTT and the duality relation of Sects. 3 and 4 for actual one-loop computations in covariant gauges $^{\S}$ requires a proper treatment of the contributions from this type of second-order poles. This point is not pursued any further in the present paper.

In physical gauges, the typical form of the gauge-mode propagator is

$$
G_{G}(q)=\frac{1}{(n \cdot q)^{k}}, \quad k=1 \text { or } 2
$$

where $n^{\mu}$ denotes an auxiliary gauge vector. In Coulomb gauge, we have $n_{\mu}=(0, \mathbf{q})$, where $\mathbf{q}$ is the space component of the gauge boson momentum $q_{\mu}=\left(q_{0}, \mathbf{q}\right)$. In axial $(n \cdot A=0)$ or planar gauges, $n^{\mu}$ is a fixed external vector. We see that $G_{G}(q)$ leads to a (first or second-order) pole when $n \cdot q=0$. Actually, in the case of axial and planar gauges, this pole has to be regularized according to a proper prescription (the precise position of the pole has to be specified by some imaginary displacement from the real axis). We do not specify the regularization prescription, since its specific form has no effect on the discussion that follows.

We now consider a generic one-loop integral, whose integrand contains gauge-mode propagators in addition to Feynman propagators. To derive a duality relation by using the residue theorem in the complex plane of the variable $q_{0}$ (as in Sect. 4), we have to take into account the possible contributions from the poles of the gauge-mode propagators.

In Coulomb gauge, the pole of $G_{G}(q)$ is located at $\mathbf{q}^{2}=0$. Applying the residue theorem in the $q_{0}$ plane at fixed values of $\mathbf{q}$ (see Sect. 4 and Appendix A), the gauge pole does not contribute. We conclude that the gauge-mode propagator remains untouched in going from the one-loop integral to its representation as a single-cut dual integral. Note, however, that this conclusion follows from having kept $\mathbf{q}$ fixed while performing the integration over $q_{0}$. Therefore, the auxiliary future-like vector $\eta^{\mu}$ of the duality relation is necessarily fixed (see Appendix A) to be $\eta_{\mu}=\left(\eta_{0}, \mathbf{0}\right)$, i.e. aligned along the time direction.

In axial or planar gauges, the pole of $G_{G}(q)$ is located at $n q=n_{0} q_{0}-n_{d-1} q_{d-1}=0$. Without loosing any generality, we can assume $n_{\mu}=\left(n_{0}, \mathbf{0}_{\perp}, n_{d-1}\right)$. We can then apply (see Sect. 4) the residue theorem in the complex plane $q_{0}$ at fixed values of the coordinates $\mathbf{q}_{\perp}$ and $q_{d-1}^{\prime}=q_{d-1}-q_{0} \eta_{d-1} / \eta_{0}$. Setting $\eta_{d-1} / \eta_{0}=n_{0} / n_{d-1}$, we have $n q=-n_{d-1} q_{d-1}^{\prime}$. Hence, $G_{G}(q)$ does not depend on the integration variable $q_{0}$. We conclude that the gauge-mode propagator, including the regularization prescription of its gauge pole, is untouched in going from the one-loop integral to its representation as a single-cut dual integral. Note, however, that we have set $\eta_{d-1} / \eta_{0}=n_{0} / n_{d-1}$. Therefore, since the vector $\eta^{\mu}$ that specifies the dual prescription is future-like, the above conclusion is valid only if the gauge vector $n^{\mu}$ is either space-like or light-like $\left(n^{2} \leq 0\right)$ and, moreover, the dual vector is fixed to be orthogonal to

\footnotetext{
$\S$ Of course, this sentence does not apply to the 't Hooft-Feynman gauge, where $G_{G}(q)$ is absent.
} 
the gauge vector (i.e. $n \cdot \eta=0$ ). These requirements are not fulfilled if $n^{\mu}$ is time-like . The derivation of a duality relation in time-like gauges requires the proper introduction of contributions from cuts of the gauge-polarization tensors (these contributions depend on the specific regularization of the gauge poles): this derivation is beyond the scope of the present paper.

Our discussion and conclusions on the duality relation in physical gauges can straightforwardly be used to draw similar conclusions on the validity of the FTT. The only difference is that, using the FTT, there is no auxiliary dual vector $\eta^{\mu}$. To be precise, in Coulomb gauge and in space-like or light-like gauges, the FTT is valid in his customary form, without introducing any multiple-cut contributions due to the gauge-polarization tensors. In time-like gauges, the poles of the gauge-polarization tensors can play a role, and their effect has to be taken into account when applying the FTT.

\section{Loop-tree duality at the amplitude level}

In the final part of Sect. 3, we have discussed how the FTT can be extended from the evaluation of the basic one-loop integrals $L^{(N)}$ to the evaluation of complete one-loop quantities (such as Green's functions and scattering amplitudes). The same type of discussion (see also Sects. 8 and 9) and analogous conclusions apply to the extension of the duality relation to the amplitude level.

The analogue of Eq. (25) is the following duality relation:

$$
\mathcal{A}^{(1-\text { loop })}=-\widetilde{\mathcal{A}}^{(1-\text { loop })}
$$

where $\mathcal{A}^{(1-\text { loop })}$ generically denotes a one-loop quantity. The expression $\widetilde{\mathcal{A}}^{(1-\text { loop })}$ on the right-hand side of Eq. (101) is obtained in the same way as $\widetilde{L}^{(N)}$ in Eqs. (32) and (33). To be precise, we start from any Feynman diagram in $\mathcal{A}^{(1-\mathrm{loop})}$ and we consider all possible replacements of each Feynman propagator $G\left(q_{i}\right)$ of its loop internal lines with the cut propagator $\widetilde{\delta}\left(q_{i} ; M_{i}\right)$; the uncut Feynman propagators in the loop are then replaced by corresponding dual propagators.

The duality relation (101) is valid in any field theory that is unitary and local. We only need to add some words of caution (see the conclusions of Sect. 9) about its applicability to theories with local gauge symmetries. In spontaneously-broken gauge theories, the duality relation is valid in the 't Hooft-Feynman gauge and in the unitary gauge. In unbroken gauge theories, the duality relation is valid in the 't Hooft-Feynman gauge; it is also valid in physical gauges specified by a gauge vector $n^{\nu}$, provided the auxiliary duality vector $\eta^{\mu}$ is chosen such that $n \cdot \eta=0$ (this excludes gauges where $n^{\nu}$ is time-like).

Equation (101) establishes a correspondence between one-loop Feynman diagrams and the phase-space integral of tree-level Feynman diagrams. The right-hand side of Eq. (101)

\footnotetext{
IFor example, in the axial gauge $A_{0}=0$, we have $n q=n_{0} q_{0}$, and the pole of the gauge-mode propagator does not decouple from the integration over $q_{0}$.
} 
can be written in the following sketchy form:

$$
\mathcal{A}^{(1-\text { loop })} \sim \int_{q} \sum_{P} \widetilde{\delta}\left(q ; M_{P}\right) \sum_{\text {d.o.f. }(P)} \mathcal{A}_{P}^{\text {(tree) }},
$$

where $\sum_{P}$ denotes the sum over the particles that can propagate in the loop internal line that have been cut, and $\sum_{\text {d.o.f.(P) }}$ denotes the sum over the degrees of freedom (such as spin, colors, ..) of the particle $P$. The integrand $\mathcal{A}_{P}^{(\text {tree })}$ is given by the sum of the tree-level Feynman diagrams that are obtained by cutting the one-loop Feynman diagrams on the left-hand side.

The structure of Eq. (101) suggests a natural question". If $\mathcal{A}^{(1-\text { loop })}$ is the one-loop expression of a specific quantity $\mathcal{A}$, is (and how is) $\mathcal{A}_{P}^{\text {(tree) }}$ related to the tree-level expression of the same quantity $\mathcal{A}$ ? In the next subsections, we show how the duality relation can be formulated directly at the amplitude level, when the quantity $\mathcal{A}$ is a Green's function. We also discuss the case of on-shell scattering amplitudes.

\subsection{Green's functions}

In the following, $\mathcal{A}_{N}\left(p_{1}, \ldots, p_{N}\right)$ denotes a generic off-shell Green's function with $N$ external lines (the outgoing momentum of the $i$-th line is $p_{i}$ ). To be precise, we consider Green's functions that are connected and amputated of the free propagators of the external lines. The tree-level and one-loop expressions of $\mathcal{A}$ are $\mathcal{A}^{\text {(tree) }}$ and $\mathcal{A}^{\text {(1-loop) }}$, respectively. The treelevel scattering amplitude for a given physical process is obtained from $\mathcal{A}^{\text {(tree) }}\left(p_{1}, \ldots, p_{N}\right)$ by setting the external momenta on their physical mass shell $\left(p_{i}^{2}=M_{i}^{2}, p_{i 0} \geq 0\right.$ for an outgoing particle, $-p_{i 0} \geq 0$ for an incoming particle) and including the appropriate wavefunction factors of the external particles. The one-loop scattering amplitude is obtained from $\mathcal{A}^{(1-\text { loop })}$ by specifying the renormalization procedure.

To simplify the illustration of the duality relation, we first consider the case with only one type of massive scalar particles. We thus refer to a theory with a single real scalar field $\phi\left(\phi^{*}=\phi\right)$ of mass $M$. The particles are self-interacting through polynomial interactions (e.g. $\phi^{3}$ or $\left.\phi^{4}\right)$. In this case, the duality relation (102) has the following explicit form:

$$
\mathcal{A}_{N}^{(1-\mathrm{loop})}\left(p_{1}, \ldots, p_{N}\right)=+\frac{1}{2} \int \frac{d^{d} q}{(2 \pi)^{d-1}} \delta_{+}\left(q^{2}-M^{2}\right) \widetilde{\mathcal{A}}_{N+2}^{\text {tree })}\left(q,-q, p_{1}, \ldots, p_{N}\right)
$$

where the integrand factor $\mathcal{A}^{\text {(tree) }}$ on the right-hand side is exactly the tree-level counterpart of the one-loop quantity $\mathcal{A}_{N}^{(1-\text { loop) }}$ on the left-hand side. The tree-level counterpart $\mathcal{A}_{N+2}^{\text {(tree) }}$ involves two additional external lines with outgoing momenta $q$ and $-q$.

The tilde superscript in $\widetilde{\mathcal{A}}^{\text {(tree) }}$ denotes the replacement of Feynman propagators with dual propagators. More precisely, to obtain $\widetilde{\mathcal{A}}^{\text {(tree) }}(q,-q, \ldots)$ from $\mathcal{A}^{\text {(tree) }}(q,-q, \ldots)$, we assign a dual propagator (rather than a Feynman propagator) to each internal line with momentum $q+k_{j}\left(k_{j}\right.$ is a linear combination of the external momenta $\left.p_{i}\right)$. We note that this

\footnotetext{
"Issues related to similar questions were discussed by Feynman [2] in the context of the FTT.
} 
step can also be performed by using a short-cut recipe: we can simply apply the momentum shift $q^{\mu} \rightarrow q^{\mu}-i 0 \eta^{\mu} /(2 \eta q)$ in the Feynman propagators of $\mathcal{A}^{\text {(tree) }}(q,-q, \ldots)$.

The momenta $q$ and $-q$ of the two additional external lines of $\mathcal{A}_{N+2}^{(\text {tree })}(q,-q, \ldots)$ in Eq. (103)are on their physical mass-shell: in this respect, $\mathcal{A}_{N+2}^{\text {(tree) }}$ is a scattering amplitude (there are no wave-function factors for scalar particles). More precisely, $\widetilde{\mathcal{A}}_{N+2}^{\text {(tree) }}(q,-q, \ldots)$ is the tree-level physical amplitude that corresponds to the forward-scattering process of a particle with momentum $q$ in the external field produced by $N$ self-interacting sources (the $N$ external legs).

In a theory with different types, $P$, of particles and antiparticles, the generalization of Eq. (103) is obtained by including a sum over the particle types. We find:

$$
\mathcal{A}_{N}^{(1-\mathrm{loop})}(\ldots)=+\frac{1}{2} \int \frac{d^{d} q}{(2 \pi)^{d-1}} \sum_{P} \delta_{+}\left(q^{2}-M_{P}^{2}\right) \sigma(P) \widetilde{\mathcal{A}}_{N+2}^{\text {tree })}(P(q) \leftarrow P(q), \ldots),
$$

where the momenta $p_{i}$ of $N$ external legs are denoted by 'dots', since they play no active role on both sides of the equation. Note that $\sum_{P}$ includes the sum over both particles and antiparticles (if $P \neq \bar{P}$ ).

The coefficient $\sigma(P)$ on the right-hand side of Eq. (104) is a Bose-Fermi statistics factor: $\sigma(P)=+1$ if $P$ is a bosonic particle (e.g. spin 0 Higgs boson, spin 1 gauge boson), and $\sigma(P)=-1$ if $P$ is a fermionic particle (e.g. spin 1/2 fermion, Faddeev-Popov ghost).

The tree-level expression $\mathcal{A}_{N+2}^{(\text {tree })}(P(q) \leftarrow P(q), \ldots)$ is the amplitude for the forwardscattering process $P(q) \rightarrow P(q)$ in the field of the $N$ external legs. It can be written as

$$
\mathcal{A}_{N+2}^{\text {(tree) }}(P(q) \leftarrow P(q), \ldots)=\sum_{\text {spin, color, .. }}\left\langle P(q)\left|\mathcal{A}_{N+2}^{(\text {tree })}(P(q), \bar{P}(-q), \ldots)\right| P(q)\right\rangle,
$$

where the ('ket' and 'bra') vectors $|P(q)\rangle$ and $\langle P(q)|$ generically denote the (spin-dependent, color-dependent, ...) wave-function factors of the forward-scattered particle $P$.

We illustrate the general notation in Eq. (105) with a few explicit examples:

- $P=$ gluon $(\lambda$ labels the spin-polarization or helicity states; $\mu, \nu$ are Lorentz indices; $a, b$ are color indices)

$$
\begin{aligned}
\mathcal{A}_{N+2}^{(\text {tree })}(g(q) \leftarrow g(q), \ldots) & =\sum_{\lambda} \sum_{\mu, \nu} \sum_{a, b}\left(\varepsilon_{\mu}^{(\lambda)}(q)\right)^{*}\left[\mathcal{A}_{N+2}^{(\text {tree }}(g(q), g(-q), \ldots)\right]_{a b}^{\mu \nu} \varepsilon_{\nu}^{(\lambda)}(q) \\
& =\sum_{\mu, \nu} d_{\nu \mu}(q) \sum_{a, b}\left[\mathcal{A}_{N+2}^{(\text {tree })}(g(q), g(-q), \ldots)\right]_{a b}^{\mu \nu},
\end{aligned}
$$

where $\varepsilon_{\nu}^{(\lambda)}(q)$ is the gluon-polarization vector; 
- $P=$ massive quark ( $s$ labels the spin; $\alpha, \beta$ are Dirac indices; $i, j$ are color indices)

$$
\begin{aligned}
\mathcal{A}_{N+2}^{(\text {tree })}(Q(q) \leftarrow Q(q), \ldots) & =\sum_{s=1,2} \sum_{\alpha, \beta} \sum_{i, j} \bar{u}_{\alpha}^{(s)}(q)\left[\mathcal{A}_{N+2}^{(\text {tree })}(Q(q), \bar{Q}(-q), \ldots)\right]_{\alpha \beta}^{i j} u_{\beta}^{(s)}(q) \\
& =\operatorname{Tr}\left[(\phi+M) \sum_{i, j}\left[\mathcal{A}_{N+2}^{(\text {tree })}(Q(q), \bar{Q}(-q), \ldots)\right]^{i j}\right], \quad(107)
\end{aligned}
$$

where $u_{\beta}^{(s)}(q)$ is the customary Dirac spinor for spin $1 / 2$ fermions;

- $P=$ massive anti-quark ( $s$ labels the spin; $\alpha, \beta$ are Dirac indices; $i, j$ are color indices)

$$
\begin{aligned}
\mathcal{A}_{N+2}^{\text {(tree) }}(\bar{Q}(q) \leftarrow \bar{Q}(q), \ldots) & =-\sum_{s=1,2} \sum_{\alpha, \beta} \sum_{i, j} \bar{v}_{\alpha}^{(s)}(q)\left[\mathcal{A}_{N+2}^{\text {(tree) }}(Q(-q), \bar{Q}(q), \ldots)\right]_{\alpha \beta}^{i j} v_{\beta}^{(s)}(q) \\
& =-\operatorname{Tr}\left[(\not-M) \sum_{i, j}\left[\mathcal{A}_{N+2}^{(\text {tree })}(Q(-q), \bar{Q}(q), \ldots)\right]^{i j}\right], \quad(108)
\end{aligned}
$$

where $v_{\beta}^{(s)}(q)$ is the customary Dirac spinor for spin $1 / 2$ anti-fermions.

Note that, as stated below Eq. (104), when considering particle-antiparticle fields we sum over both particles and antiparticles. However, on the right-hand side of Eq. (104), $\sum_{P}$ can equivalently be defined to mean just the sum over particles. According to this alternative definition, the antiparticle contribution $\widetilde{\mathcal{A}}_{N+2}^{\text {(tree) }}(\bar{P}(q) \leftarrow \bar{P}(q), \ldots)$ is absent, and the corresponding particle contribution $\widetilde{\mathcal{A}}_{N+2}^{\text {(tree) }}(P(q) \leftarrow P(q), \ldots)$ must be multiplied by a factor of 2 .

\subsection{Scattering amplitudes}

To extend the discussion of Sect. 10.1 to scattering amplitudes, the only relevant point to be examined is the on-shell limit of the corresponding Green's functions (the introduction of the wave-function factors of the external lines is straightforward).

Considering the off-shell Green's function $\mathcal{A}_{N}^{(1-\text { loop })}$, we introduce the following decomposition:

$$
\mathcal{A}_{N}^{(1-\text { loop })}=\mathcal{A}_{N}^{(1-\text { loop; ex. })}+\mathcal{A}_{N}^{(1-\text { loop; in. })},
$$

where $\mathcal{A}_{N}^{(1-\text { loop; ex.) }}$ is the contribution from one-loop insertions on the $N$ external lines, while $\mathcal{A}_{N}^{(1-\text { loop; in.) }}$ is the remaining contribution (i.e. one-loop insertions on internal lines). In explicit form, we have

$$
\mathcal{A}_{N}^{(1-\text { loop } ; \text { ex. })}\left(p_{1}, \ldots, p_{N}\right)=\sum_{j=1}^{N} \mathcal{A}_{2}^{(1-\text { loop })}\left(p_{j},-p_{j}\right) \frac{i D_{j}\left(p_{j}\right)}{p_{j}^{2}-M_{j}^{2}+i 0} \mathcal{A}_{N}^{\text {(tree) }}\left(p_{1}, \ldots, p_{N}\right)
$$

where $D_{j}\left(p_{j}\right)$ is the spin-polarization factor of the particle in the internal line with momentum $p_{j}$. 
As is well known, $\mathcal{A}_{N}^{(1-\text { loop; ex.) }}$ cannot directly be evaluated on-shell, because of the ensuing kinematical singularity from its external-line propagators (the propagators with momentum $p_{j}$ in Eq. (110)). Thus, to calculate the one-loop scattering amplitude, $\mathcal{A}_{N}^{(1-\text { loop; ex. })}$ has to be first evaluated off-shell, then it has to be renormalized (mass and wave-function renormalization), before considering its on-shell limit.

In contrast, the one-loop contribution $\mathcal{A}_{N}^{(1-\text { loop; in.) }}$ can directly be computed in the onshell limit. In particular, we can write a duality relation in the form of Eq. (101):

$$
\mathcal{A}_{N}^{(1-\text { loop; in. })}=-\widetilde{\mathcal{A}}_{N}^{(1-\text { loop; in. })} .
$$

Here, the integrand of the phase-space integral on the right-hand side contains a sum of on-shell tree-level Feynman diagrams (the $N$ external lines are on-shell, and the two additional lines from cutting the loop are also on-shell). The algebraic computation of the integrand is thus completely analogous to the computation of the (on-shell) tree-level scattering amplitude with $N+2$ external legs. Having performed the tree-level computation of the integrand, the result can be integrated over the single-particle phase-space to obtain the full one-loop term $\mathcal{A}_{N}^{(1-\text { loop; in.) }}$.

We point out that the integrand of the phase-space integral on the right-hand side of Eq. (111) is not equal (modulo the replacement of Feynman with dual propagators) to the tree-level scattering amplitude with $N+2$ external legs. This is because a subset of the diagrams that enter the complete tree-level scattering amplitude is not included. This subset has been removed by considering only $\mathcal{A}_{N}^{(1-\text { loop; in. })}$, i.e. by removing $\mathcal{A}_{N}^{(1-\text { loop; ex. })}$ from the complete one-loop expression $\mathcal{A}_{N}^{(1-\text { loop })}$.

This 'missing' subset of tree-level diagrams can be reinserted in the duality relation. However, as discussed below, this makes more delicate the on-shell limit.

We consider the internal-line contribution $\mathcal{A}_{N}^{(1-\text { loop; in.) }}$ before setting the external lines on-shell. We can write the following duality relation:

$$
\begin{aligned}
\mathcal{A}_{N}^{(1-\text { loop; in. })}\left(p_{1}, \ldots, p_{N}\right)=+\frac{1}{2} \int \frac{d^{d} q}{(2 \pi)^{d-1}} \sum_{P} \delta_{+}\left(q^{2}-M_{P}^{2}\right) \sigma(P) \\
\quad \times\left\{\widetilde{\mathcal{A}}_{N+2}^{\text {tree })}\left(P(q) \leftarrow P(q), p_{1}, \ldots, p_{N}\right)\right. \\
\left.\quad-\sum_{j=1}^{N} \widetilde{\mathcal{A}}_{4}^{\text {(tree })}\left(P(q) \leftarrow P(q), p_{j},-p_{j}\right) \frac{i D_{j}\left(p_{j}\right)}{p_{j}^{2}-M_{j}^{2}+i 0} \mathcal{A}_{N}^{\text {(tree })}\left(p_{1}, \ldots, p_{N}\right)\right\} .
\end{aligned}
$$

The derivation of this equation is simple. We first use Eq. (109) to express $\mathcal{A}_{N}^{(1-\text { loop; in.) }}$ as difference of $\mathcal{A}_{N}^{(1-\text { loop })}$ and $\mathcal{A}_{N}^{(1-\text { loop; ex. })}$. Then we use Eq. (110) to rewrite $\mathcal{A}_{N}^{(1-\text { loop; ex. })}$ in terms of $\mathcal{A}_{2}^{(1-\text { loop })}$. Finally, we express the full one-loop Green's functions $\mathcal{A}_{N}^{(1-\text { loop })}$ and $\mathcal{A}_{2}^{(1-\text { loop })}$ in terms of the duality relation (104).

The duality relation (112) involves the phase-space integration of complete tree-level Green's functions, namely $\mathcal{A}_{N}^{\text {(tree) }}\left(p_{1}, \ldots, p_{N}\right)$, and (the duality-propagator version of) $\mathcal{A}_{N+2}^{\text {(tree) }}\left(P(q) \leftarrow P(q), p_{1}, \ldots, p_{N}\right)$ and $\mathcal{A}_{4}^{\text {(tree) }}\left(P(q) \leftarrow P(q), p_{j},-p_{j}\right)$. The integrand factor in the curly bracket on the right-hand side is well defined in the on-shell limit. However, the 
two terms in the curly bracket are separately singular in the on-shell limit. The singularity is purely kinematical; it simply arises from the propagators of the lines with momenta equal to the momenta $p_{j}$ of the external lines. Various procedures can be devised to introduce an intermediate regularization of the separate singularities, so as to directly evaluate the two terms close to on-shell kinematical configurations.

\section{Final remarks}

Applying directly the Cauchy residue theorem in the complex plane of any of the spacetime coordinates of the loop momentum we have derived a duality relation between one-loop integrals and single-cut phase-space integrals. The calculation of the residues is elementary, but introduces several subtleties. The location in the complex plane of the pole of the cut propagator modifies the original $+i 0$ Feynman prescription of the uncut propagators. Oneloop integrals are then written as a linear combination of $N$ single-cut phase-space integrals, with propagators regularized by a new complex Lorentz-covariant prescription, named dual prescription. It is defined through a future-like auxiliary vector $\eta$. This simple replacement compensates for the absence of multiple-cut contributions that appear in the FTT. The dependence on $\eta$ cancels, as expected, in the sum of all the single-cut contributions, leading to $\eta$-independent results.

We have generalized the duality relation for internal massive propagators and unstable particles. Real masses just modify the position of the poles in the complex plane by a translation parallel to the real axis, and thus do not affect the dual prescription. Unstable particles introduce a finite imaginary contribution in their propagators. The poles of the complex mass propagators are located at a finite imaginary distance from the real axis, and the $+i 0$ prescription of the usual Feynman propagators can be removed when propagators of unstable particles are cut.

Particular care has to be taken with gauge propagators in both the FTT and the duality relation due to the presence of unphysical extra gauge poles. We have discussed this issue, and have identified the different gauge choices where the duality relation can be applied in its simpler form.

Finally, we have extended the duality relation from Feynman integrals to Green's functions and scattering amplitudes. One-loop scattering amplitudes can be obtained starting from tree-level scattering amplitudes (or, more precisely, from Feynman diagrams that enter the computation of tree-level scattering amplitudes), where (some of) the internal propagators are replaced by dual propagators. This tree-level counterpart is then integrated over a single-particle phase space to get the one-loop scattering amplitude. Since efficient methods for the numerical calculation of tree-level amplitudes exist and have been automated, the duality relation is also suitable for automation of the numerical evaluation of one-loop amplitudes. The numerical evaluation can also be extended to the level of physical cross-sections at next-to-leading order [3, 5], since the single-particle phase-space integration of the duality relation can directly be combined with the integration over the multi-particle phase-space of the physical process. The duality relation can also be used to obtain one-loop results in analytic form, starting from corresponding tree-level results. 
The extension of the duality relation from one-loop to two-loop Feynman diagrams is under investigation [5].

\section{Acknowledgements}

Financial support by the Ministerio de Educación y Ciencia (MEC) under grants FPA200760323 and CPAN (CSD2007-00042), by the European Commission under contracts FLAVIAnet (MRTN-CT-2006-035482), HEPTOOLS (MRTN-CT-2006-035505), and MCnet (MRTNCT-2006-035606), by the INFN-MEC agreement and by BMBF is gratefully acknowledged.

Major fractions of the work have been completed while three of us (S.C., F.K., G.R.) were participating in the Workshop Advancing Collider Physics: from Twistors to Monte Carlos at the Galileo Galilei Institute (GGI) for Theoretical Physics in Florence: we wish to thank the GGI for its hospitality and the INFN for partial support.

\section{A Appendix: Derivation of the duality relation}

In Sect. 4 we have illustrated how the duality relation in Eqs. (32) and (33) can be derived by using the residue theorem. The derivation is simple. However, it involves some subtle points. These points are discussed in detail in this Appendix.

Applying the residue theorem in the complex plane of the variable $q_{0}$, the computation of the one-loop integral $L^{(N)}$ reduces to the evaluation of the residues at $N$ poles, according to Eqs. (27) and (28).

The evaluation of the residues in Eq. (28) is a key point in the derivation of the duality

relation. To make this point as clear as possible, we first introduce the notation $q_{i 0}^{(+)}$to explicitly denote the location of the $i$-th pole, i.e. the location of the pole with negative imaginary part (see Eq. (12)) that is produced by the propagator $G\left(q_{i}\right)$. Then, we further simplify our notation with respect to the explicit dependence on the subscripts that label the momenta. We notice that we can write $G\left(q_{j}\right)=G\left(q_{i}+\left(q_{j}-q_{i}\right)\right)$, where $q_{i}$ depends on the loop momentum while $\left(q_{j}-q_{i}\right)=k_{j i}$ is a linear combination of the external momenta (see Eq. (2)). Therefore, to carry out the explicit computation the $i$-th residue in Eq. (28), we re-label the momenta as $q_{i} \rightarrow q$ and $q_{j} \rightarrow q+k_{j}$, and we simply evaluate the term

$$
\left[\operatorname{Res}_{\left\{q_{0}=q_{0}^{(+)}\right\}} G(q)\right]\left[\prod_{j} G\left(q+k_{j}\right)\right]_{q_{0}=q_{0}^{(+)}}
$$

where (see Eq. (12))

$$
q_{0}^{(+)}=\sqrt{\mathbf{q}^{2}-i 0} .
$$

In the next paragraphs, we follow the steps of Sect. 4 (see Eqs. (29) and (30)) and we separately compute the residue of $G(q)$ and and its prefactor, the associated factor coming from the propagators $G\left(q+k_{j}\right)$. 
The computation of the residue of $G(q)$ gives

$$
\begin{aligned}
\operatorname{Res}_{\left\{q_{0}=q_{0}^{(+)}\right\}} G(q) & =\lim _{q_{0} \rightarrow q_{0}^{(+)}}\left\{\left(q_{0}-q_{0}^{(+)}\right) \frac{1}{q_{0}^{2}-\mathbf{q}^{2}+i 0}\right\}=\frac{1}{2 q_{0}^{(+)}} \\
& =\frac{1}{2 \sqrt{\mathbf{q}^{2}}}=\int d q_{0} \delta_{+}\left(q^{2}\right)
\end{aligned}
$$

thus leading to the result in Eq. (29). Note that the first equality in the second line of Eq. (115) is obtained by removing the $i 0$ prescription from the previous expression. This is fully justified. Indeed, the term $\left(q_{0}^{(+)}\right)^{-1}=\left(\sqrt{\mathbf{q}^{2}-i 0}\right)^{-1}$ becomes singular only when $\mathbf{q}^{2} \rightarrow 0$, and this corresponds to an end-point singularity in the integration over $\mathbf{q}$ : therefore the $i 0$ prescription has no regularization effect on such end-point singularity. The second equality in the second line of Eq. (115) simply follows from the definition of the on-shell delta function $\delta_{+}\left(q^{2}\right)$.

We now consider the evaluation of the residue prefactor (the second square-bracket factor in Eq. (113)). We first recall that the $i 0$ prescription of the Feynman propagators has played an important role in the application (see Eqs. (27) and (113)) of the residue theorem to the computation of the loop integral: having it selected the pole with negative imaginary part, $q_{0}=q_{0}^{(+)}$, the prescription has eventually singled out (see Eq. (115)) the on-shell mode with positive definite energy, $q_{0}=|\mathbf{q}|$. However, we can observe that the result in Eq. (115) can be obtained by removing (neglecting) the $i 0$ prescription either in $q_{0}^{(+)}\left(q_{0}^{(+)} \rightarrow|\mathbf{q}|\right)$ or in $G(q)\left(G(q) \rightarrow 1 / q^{2}\right)$ :

$$
\operatorname{Res}_{\left\{q_{0}=q_{0}^{(+)}\right\}} G(q)=\operatorname{Res}_{\left\{q_{0}=|\mathbf{q}|\right\}} \frac{1}{q^{2}}=\int d q_{0} \delta_{+}\left(q^{2}\right) .
$$

Therefore, the $i 0$ prescription has no effect on the actual calculation of the residue of the propagator $G(q)$ in Eq. (113). On the basis of this observation, we might conclude that the $i 0$ prescription has also no effect on the calculation of the residue prefactor in Eq. (113), since the propagators $G\left(q+k_{j}\right)$ are not singular when evaluated at the poles of $G(q)$. We might thus compute the residue prefactor by removing the $i 0$ prescription; we would obtain

$$
\left[\prod_{j} G\left(q+k_{j}\right)\right]_{q_{0}=q_{0}^{(+)}} \rightarrow\left[\prod_{j} \frac{1}{\left(q+k_{j}\right)^{2}}\right]_{q_{0}=|\mathbf{q}|} .
$$

The expression on the right-hand side of Eq. (117) is well-defined, but, when it is inserted (through Eqs. (113) and (28)) in Eq. (27), it leads to an ill-defined result: the integration over $\mathbf{q}$ is singular at any phase-space points where the denominator factors $\left(q+k_{j}\right)^{2}$ vanish. To recover a well-defined result, we have to reintroduce the $i 0$ prescription in the residue prefactor. We might thus maintain the $i 0$ prescription in the Feynman propagators $G\left(q+k_{j}\right)$ and still keep $q_{0}$ at its on-shell value $q_{0}=|\mathbf{q}|$; we would obtain

$$
\left[\prod_{j} G\left(q+k_{j}\right)\right]_{q_{0}=q_{0}^{(+)}} \rightarrow\left[\prod_{j} \frac{1}{\left(q+k_{j}\right)^{2}+i 0}\right]_{q_{0}=|\mathbf{q}|} .
$$

Inserting (through Eqs. (113) and (28)) Eq. (115) and the right-hand side of Eq. (118) in Eq. (27), we obtain a well-defined result for the one-loop integral, since the singularities 
from the propagators $1 /\left(q+k_{j}\right)^{2}$ are now regularized by the Feynman $i 0$ prescription. However, this result for the one-loop integral would be exactly equal (see Eqs. (20) and $(22))$ to the sole 1 -cut contribution, $L_{1-\text { cut }}$, of the FTT. The ensuing contradiction with the FTT could be removed only if the total contribution, $L_{2-\text { cut }}+L_{3-\text { cut }}+\ldots$, to the FTT from multiple cuts vanishes; this is obviously unlikely, and it is actually not true as shown by the explicit one-loop calculations performed in Sect. 5 .

The discussion of the previous paragraph illustrates that the evaluation of the oneloop integrals by the direct application of the residue theorem (as in Eq. (27)) involves some subtleties. The subtleties mainly concern the correct treatment of the Feynman $i 0$ prescription in the calculation of the residue prefactors. A consistent treatment requires the strict computation of the residue prefactor in Eq. (113): the $i 0$ prescription in both $G\left(q+k_{j}\right)$ and $q_{0}^{(+)}$has to be dealt with by considering the imaginary part $i 0$ as a finite (thus, for instance, $2 i 0 \neq i 0$ ), though possibly small, quantity; the limit of infinitesimal values of $i 0$ has to be taken only at the very end of the computation, thus leading to the interpretation of the ensuing $i 0$ prescription as mathematical distribution. Applying this strict procedure, we obtain

$$
\begin{aligned}
& {\left[\prod_{j} G\left(q+k_{j}\right)\right]_{q_{0}=q_{0}^{(+)}}=\left[\prod_{j} \frac{1}{\left(q+k_{j}\right)^{2}+i 0}\right]_{q_{0}=q_{0}^{(+)}}=\prod_{j} \frac{1}{2 q_{0}^{(+)} k_{j 0}-2 \mathbf{q} \cdot \mathbf{k}_{j}+k_{j}^{2}}} \\
& \quad=\prod_{j} \frac{1}{2|\mathbf{q}| k_{j 0}-2 \mathbf{q} \cdot \mathbf{k}_{j}+k_{j}^{2}-i 0 k_{j 0} /|\mathbf{q}|}=\left[\prod_{j} \frac{1}{2 q k_{j}+k_{j}^{2}-i 0 k_{j 0} / q_{0}}\right]_{q_{0}=|\mathbf{q}|} .(119)
\end{aligned}
$$

The last equality on the first line of Eq. (119) simply follows by setting $q_{0}=q_{0}^{(+)}$in the expression on the square bracket (note, in particular, that $q^{2}=-i 0$ ). Then, the first equality on the second line follows from $2 q_{0}^{(+)} \simeq 2|\mathbf{q}|-i 0 /|\mathbf{q}|$ (i.e. from expanding $q_{0}^{(+)}$at small values of $i 0)$.

The result in Eq. (119) for the residue prefactor is well-defined and leads to a well-defined (i.e. non singular) expression when it is inserted in Eq. (27). Indeed, the possible singularities from each of the propagators $1 /\left(q+k_{j}\right)^{2}$ are regularized by the displacement produced by the associated imaginary amount $i 0 k_{j 0} / q_{0}$. Performing the limit of infinitesimal values of $i 0$, only the sign of the $i 0$ prescription (and not its actual size) is relevant. Therefore, since $q_{0}$ is positive, in Eq. (119) we can perform the replacement $i 0 k_{j 0} / q_{0} \rightarrow i 0 \eta k_{j}$, where $\eta^{\mu}$ is the vector $\eta^{\mu}=\left(\eta_{0}, \mathbf{0}\right)$ with $\eta_{0}>0$; we finally obtain

$$
\left[\prod_{j} G\left(q+k_{j}\right)\right]_{q_{0}=q_{0}^{(+)}}=\left[\prod_{j} \frac{1}{\left(q+k_{j}\right)^{2}-i 0 \eta k_{j}}\right]_{q_{0}=|\mathbf{q}|}
$$

which is the result in Eq. (30). Actually, to be precise, Eq. (30) is recovered by reintroducing the original labels of the momenta of the loop integral according to the replacements $q \rightarrow q_{i}$, $k_{j} \rightarrow q_{j}-q_{i}$ (see the discussion above Eq. (113)).

In the following we explain in more detail the origin of the $\eta$ dependence in the $i 0$ prescription of the dual propagators. The explicit calculation performed in this Appendix leads to the introduction of the future-like vector $\eta^{\mu}=\left(\eta_{0}, \mathbf{0}\right)$ (see Eqs. (119) and (120)). As 
discussed in Sect. 4, different future-like vectors can be introduced by applying the residue theorem in different systems of coordinates. To clarify this point, we explicitly show the application of the residue theorem in light-cone coordinates.

Using light-cone coordinates (see Eq. (6)) rather than space-time coordinates (as in Eq. (27)), the one-loop integral can be evaluated as follows:

$$
\begin{aligned}
L^{(N)}\left(p_{1}, p_{2}, \ldots, p_{N}\right) & =\int_{\left(q_{-}, \mathbf{q}_{\perp}\right)} \int d q_{+} \prod_{i=1}^{N} G\left(q_{i}\right) \\
& =-2 \pi i \int_{\left(q_{-}, \mathbf{q}_{\perp}\right)} \sum \operatorname{Res}_{\left\{\operatorname{Im} q_{+}<0\right\}}\left[\prod_{i=1}^{N} G\left(q_{i}\right)\right]
\end{aligned}
$$

where we have applied the residue theorem by closing the integration contour at $\infty$ in the lower half-plane of the complex variable $q_{+}$(see Figs. 2 and 3). We can now compute the residues in Eq. (121) by closely following the analogous computation in Eqs. (113), (115) and (119).

The analogue of the term in Eq. (113) is

$$
\left[\operatorname{Res}_{\left\{q_{+}=q_{+}^{(+)}\right\}} G(q)\right]\left[\prod_{j} G\left(q+k_{j}\right)\right]_{q_{+}=q_{+}^{(+)}},
$$

where $q_{+}^{(+)}$denotes the location (in the $q_{+}$plane) of the pole with negative imaginary part that is produced by the propagator $G(q)$. Thus (see Eq. (12)), we have

$$
q_{+}^{(+)}=\frac{\mathbf{q}_{\perp}^{2}-i 0}{2 q_{-}}, \text {with } q_{-}>0
$$

where the requirement of negative imaginary part leads to the constraint $q_{-}>0$.

The computation of the residue of $G(q)$ gives

$$
\begin{aligned}
\operatorname{Res}_{\left\{q_{+}=q_{+}^{(+)}\right\}} G(q) & =\theta\left(q_{-}\right) \lim _{q_{+} \rightarrow q_{+}^{(+)}}\left\{\left(q_{+}-q_{+}^{(+)}\right) \frac{1}{2 q_{+} q_{-}-\mathbf{q}_{\perp}^{2}+i 0}\right\} \\
& =\theta\left(q_{-}\right) \frac{1}{2 q_{-}}=\int d q_{+} \delta_{+}\left(q^{2}\right) .
\end{aligned}
$$

We see that the residue produces the same factor as in Eq. (115).

The residue prefactor is evaluated by using the same procedure as in Eqs. (119) and (120). We obtain

$$
\begin{aligned}
& {\left[\prod_{j} G\left(q+k_{j}\right)\right]_{q_{+}=q_{+}^{(+)}}=\prod_{j} \frac{1}{2 q_{+}^{(+)} k_{j-}+2 q_{-} k_{j+}-2 \mathbf{q}_{\perp} \cdot \mathbf{k}_{\perp j}+k_{j}^{2}}} \\
& \quad=\left[\prod_{j} \frac{1}{2 q k_{j}+k_{j}^{2}-i 0 k_{j-} / q_{-}}\right]_{q_{+}=\mathbf{q}_{\perp}{ }^{2} / q_{-}}=\left[\prod_{j} \frac{1}{\left(q+k_{j}\right)^{2}-i 0 \eta k_{j}}\right]_{q_{+}=\mathbf{q}_{\perp}{ }^{2} / q_{-}} .
\end{aligned}
$$


The last equality in this equation has been obtained by performing the limit of infinitesimal values of $i 0$, analogously to Eq. (120). Since $q_{-}$is positive, we have thus implemented the replacement $i 0 k_{j-} / q_{-} \rightarrow i 0 \eta k_{j}$ where, in the present case, we have introduced the futurelike vector $\eta^{\mu}=\left(\eta_{+}, \mathbf{0}_{\perp}, \eta_{-}=0\right)$ with $\eta_{+}=\eta_{0} \sqrt{2}>0$.

It is important to note that, owing to the on-shell condition $\delta_{+}\left(q^{2}\right)$, Eqs. (120) and (125) have the same form. However, the corresponding auxiliary vectors $\eta^{\mu}$ are different. Though $\eta_{0}>0$ in both equations, in Eq. (120) $\eta$ is time-like $\left(\eta^{2}>0\right)$, whereas in Eq. (125) $\eta$ is light-like $\left(\eta^{2}=0\right)$.

We also note that the use of the residue theorem in the complex plane $q_{0}$ at fixed values of $q_{-}$and $\mathbf{q}_{\perp}$ would lead to a residue prefactor with exactly the same light-like vector $\eta^{\mu}$ as in Eq. (125).

The main features of the calculation presented in this Appendix are very general: they are valid in any system of coordinates that can be used to apply the residue theorem. The residue of $G(q)$ always replaces the Feynman propagator with the corresponding on-shell propagator $\delta_{+}\left(q^{2}\right)$ (see Eqs. (29), (115) and (124)); the residue prefactor produces dual propagators with an auxiliary vector $\eta$ that depends on the specific system of coordinates that has been actually used (see Eqs. (30), (120) and (125)).

We conclude this Appendix by briefly describing the derivation (through the residue theorem) of the generalized duality relation in Eq. (85). The generalized one-loop integral on the left-hand side contains both Feynman and advanced propagators. Before applying the residue theorem, we can specify how the infinitesimal limit ' $i 0 \rightarrow 0$ ' is performed in the two different types of propagators. We rewrite the advanced propagator as $G_{A}(q)=$ $\left[q^{2}-i \rho \operatorname{sign}\left(q_{0}\right)\right]^{-1}$ and, evaluating the one-loop integral, we perform first the limit $i 0 \rightarrow 0$ (at fixed $\rho$ ) in the Feynman propagators and then the limit $i \rho \rightarrow 0$ in the advanced propagators. When we apply the residue theorem by closing the integration contour at $\infty$ in the lower half-plane of the complex variable $q_{0}$, the poles of the advanced propagators do not contribute. Performing the limit $i 0 \rightarrow 0$, the Feynman propagators behave exactly as in the case of the duality relation in Eqs. (32) and (33), while the advanced propagators are untouched (since $\rho$ is kept finite). Finally, we perform the infinitesimal limit $i \rho \rightarrow 0$. We thus obtain Eq. (85), where the advanced propagators are unchanged in going from the one-loop integral on the left-hand side to the phase-space integral on the right-hand side.

\section{B Appendix: An algebraic relation}

This Appendix is devoted to prove the relation (71). More generally, we consider a set of $n$ real variables $\lambda_{i}$, with $i=1,2, \ldots, n$, that fulfill the constraint

$$
\sum_{i=1}^{n} \lambda_{i}=0 .
$$

Then, we shall prove the following relation:

$$
\theta\left(\lambda_{1}\right) \theta\left(\lambda_{1}+\lambda_{2}\right) \ldots \theta\left(\lambda_{1}+\lambda_{2}+\cdots+\lambda_{n-1}\right)+\text { cyclic perms. }=1 .
$$


Equation (71) simply follows by setting $\lambda_{i}=\eta p_{i}$. Note that the future-like nature of the vector $\eta$ plays no role in Eq. (71). This equation is just a consequence of momentum conservation, namely Eq. (126).

To simplify the notation we define $\theta(i, j) \equiv \theta\left(\lambda_{i}+\lambda_{i+1}+\ldots+\lambda_{j}\right)$; with $\lambda_{i}$ defined modulo $n$ : $\lambda_{n+i}=\lambda_{i}$. The relation (127) is equivalent to

$$
F_{n}\left(\lambda_{1}, \cdots, \lambda_{n}\right)=\sum_{i=1}^{n} \theta(i) \theta(i, i+1) \cdots \theta(i, i+n-2)=1 .
$$

Then, we proceed by induction. Assuming that Eq. (128) is valid for $n-1$ real variables we shall proof that it is valid for $n$.

Select the last two variables $\lambda_{n-1}$ and $\lambda_{n}$, and consider separately the two cases: $\theta(n-$ $1, n)=1$ and $\theta(n-1, n)=0$. If $\theta(n-1, n)=1$ the first term in the sum of Eq. (128) vanishes because $\theta(1, n-2)=0$ due to momentum conservation. For the second and subsequent terms and as far as $i \leq n-2$ we will have $\theta(i, n-2) \theta(i, n)=\theta(i, n-2)$. The $n-1$ term is proportional to $\theta(n-1, n)=1$ and then it is the product of $n-2$ theta functions also. Then we can apply Eq. (128) with the set of $n-1$ elements $\left\{\lambda_{1}, \cdots, \lambda_{n-2}, \lambda_{n-1}+\lambda_{n}\right\}$. Most of the terms cancel to each other by applying Eq. (128) with $n-1$ elements, and we find

$$
F_{n}\left(\lambda_{1}, \cdots, \lambda_{n}\right)=1+\theta(n, 1) \theta(n, 2) \cdots \theta(n, n-2)(\theta(n)-1) .
$$

If $\lambda_{n}$ is positive then $F_{n}=1$ as we wanted to demonstrate. If $\lambda_{n}$ is negative then $\theta(n, n-2)=$ 0 because $\lambda_{1, n-2}$ is also negative, and then $F_{n}=1$ again.

In the other case, namely for $\theta(n-1, n)=0$, the $n-1^{\text {th }}$ term of the sum in Eq. (128) obviously vanishes, and in the first term of that sum is proportional to $\theta(1, n-2)=1$. Then we apply Eq. (128) to the first term with the set of $n-1$ elements $\left\{\lambda_{1}, \cdots, \lambda_{n-2}+\lambda_{n-1}, \lambda_{n}\right\}$ and find

$$
\begin{aligned}
F_{n}\left(\lambda_{1}, \cdots, \lambda_{n}\right) & =1+\sum_{i=2}^{n-3} \theta(i) \cdots \theta(i, n-3) \theta(i, n-1) \cdots \theta(i, i+n-2)(\theta(i, n-2)-1) \\
& +\theta(n-2, n-1) \theta(n-2, n) \cdots \theta(n-2, n-4)(\theta(n-2)-1) \\
& +\theta(n) \theta(n, 1) \cdots \theta(n, n-3)(\theta(n, n-3)-1)
\end{aligned}
$$

but due to momentum conservation

$$
\begin{aligned}
& \theta(i, n)(\theta(i, n-2)-1)=-\theta(n-1, i-1) \theta(i, n)=0, \\
& \theta(n-2, n)(\theta(n-2)-1)=-\theta(n-1, n-3) \theta(n-2, n)=0, \\
& \theta(n, n-3)(\theta(n, n-3)-1)=-\theta(n, n-3) \theta(n-2, n-1)=0,
\end{aligned}
$$

and then $F_{n}=1$. This completes the proof of Eq. (128).

\section{References}

[1] R. P. Feynman, Acta Phys. Polon. 24 (1963) 697. 
[2] R. P. Feynman, Closed Loop And Tree Diagrams, in Magic Without Magic, ed. J. R. Klauder, (Freeman, San Francisco, 1972), p. 355, in Selected papers of Richard Feynman, ed. L. M. Brown (World Scientific, Singapore, 2000) p. 867.

[3] S. Catani, presented at the Workshop $H P^{2}$ : High Precision for Hard Processes at the LHC, Sept. 2006, Zurich, Switzerland (http://wwwtheorie.physik.unizh.ch/research_groups/particle/hp2/); G. Rodrigo, presented at the International Linear Collider (ILC) Workshop (ILC-ECFA and GDE Joint Meeting), Nov. 2006, Valencia, Spain (http : //ific.uv.es/ ilc/ECFA-GDE2006/); T. Gleisberg, presented at the Conference Heidelberg 07 (DPG Spring Meeting), March 2007, Heidelberg, Germany (http : //www.dpg-tagungen.de/program/heidelberg/).

[4] T. Gleisberg, PhD Thesis, University of Dresden.

[5] S. Catani, T. Gleisberg, F. Krauss, G. Rodrigo and J. Winter, in preparation.

[6] A. Brandhuber, B. Spence and G. Travaglini, JHEP 0601 (2006) 142.

[7] E. Witten, Commun. Math. Phys. 252 (2004) 189.

[8] F. Cachazo, P. Svrcek and E. Witten, JHEP 0410 (2004) 074.

[9] See Z. Bern, L. J. Dixon and D. A. Kosower, Annals Phys. 322 (2007) 1587 and references therein.

[10] A. Denner, S. Dittmaier, M. Roth and D. Wackeroth, Nucl. Phys. B 560 (1999) 33; A. Denner, S. Dittmaier, M. Roth and L. H. Wieders, Nucl. Phys. B 724 (2005) 247. 